\title{
Influence of disorder on the transport properties of heavy-fermion systems
}

\author{
Claas Grenzebach, Frithjof B. Anders, and Gerd Czycholl \\ Institut für Theoretische Physik, Universität Bremen, P.O. Box 330 440, D-28334 Bremen, Germany \\ Thomas Pruschke \\ Institut für Theoretische Physik, Universität Göttingen, D-37077 Göttingen, Germany
}

\begin{abstract}
The influence of substitutional disorder on the transport properties of heavy-fermion systems is investigated. We extend the dynamical mean-field theory treatment of the periodic Anderson model (PAM) to a coherentpotential approximation for disordered strongly correlated electron systems. Considering two distinct local environments of a binary alloy $A_{c} B_{1-c}$ with arbitrary concentration $c$, we explore two types of disorder: on the $f$ site and on the ligand sites. We calculate the spectral functions and self-energies for the disordered PAM as well as the temperature dependence of the resistivity and the thermoelectric power. The characteristic concentration dependence as well as the order of magnitude of transport properties are reproduced for metallic heavy-fermion systems and Kondo insulators. In particular, sign changes of the Seebeck coefficient as function of temperature and concentration are observed.
\end{abstract}

PACS numbers: 71.10.Fd, 71.27.+a, 72.10.-d, 72.15.-v

\section{INTRODUCTION}

Heavy-fermion systems (HFSs) are characterized by quasiparticles with a very large effective mass at low temperatures $T$. This behavior occurs in many lanthanide (rare-earth) and actinide compounds and has its origin in the hybridization between the conduction bands and the local moments of incompletely filled $f$ shells of the lanthanide or actinide ions. ${ }^{1,2}$ These $f$ electrons contribute substantially to the formation of the heavy quasiparticles. Measurements of the transport coefficients on HFSs reveal characteristic anomalies at low temperatures. The temperature dependence of the resistivity $\rho(T)$ of metallic HFSs (such as $\mathrm{CePd}_{3}, \mathrm{CeAl}_{3}, \mathrm{CeCu}_{6}, \mathrm{CeCu}_{2} \mathrm{Si}_{2}$, $\mathrm{UPd}_{2} \mathrm{Al}_{3}$, etc.; see Refs. 2,3,4,5,6,7) shows a rapid increase with increasing $T$ starting from a small residual value, which can often be fitted by a $T^{2}$ law. The resistivity saturates at $T_{\max }$ and decreases with increasing $T$ for $T>T_{\max }$ as $\rho(T) \sim \log \left(T_{\max } / T\right)$, characteristic of the Kondo effect. In Kondo insulators, $, 8,9$ however, a narrow gap opens ${ }^{10}$ at the Fermi energy at low temperatures. In such materials, e.g., $\mathrm{SmB}_{6}$ or $\mathrm{Ce}_{3} \mathrm{Bi}_{4} \mathrm{Pt}_{3}$, the resistivity shows an activation behavior for $T \rightarrow 0$. Another interesting transport quantity is the thermoelectric power or Seebeck coefficient $S(T)$. At temperatures comparable to $T_{\max }$, the thermoelectric power can reach absolut values of about $50 \mu \mathrm{V} / \mathrm{K}$, often accompanied with sign changes at intermediate temperatures. ${ }^{1,2}$ In Kondo insulators, even larger values of the thermoelectric power up to $300 \mu \mathrm{V} / \mathrm{K}$ have been observed, ${ }^{11,12}$ which may be interesting for low-temperature thermoelectric cooling.

In this paper, we examine alloys of heavy-fermion materials with arbitrary concentrations. Disorder is introduced by substitution of ligand ions (disorder on the ligand sites) or of lanthanide or actinide ions (disorder on the $f$ site). A special case of disorder on the $f$ site is given by replacing $f$-electron ions (e.g., Ce) by a certain amount of nonmagnetic impurities (e.g., La) referred to as "Kondo holes." While we focus on local disorder only, band disorder in the Kondo lattice model has been recently considered ${ }^{13}$ using a slave-boson mean-field approach.

Disorder has strong impact on the transport properties such as $\rho(T)$ and $S(T)$. In substitutional alloys such as $\mathrm{La}_{1-x} \mathrm{Ce}_{x} \mathrm{Pd}_{3},{ }^{3} \mathrm{Ce}_{x} \mathrm{La}_{1-x} \mathrm{Cu}_{6},{ }^{6} \mathrm{Ce}_{x} \mathrm{La}_{1-x} \mathrm{Cu}_{2.05} \mathrm{Si}_{2},{ }^{14,15}$ $\mathrm{U}_{1-x} \mathrm{Th}_{x} \mathrm{Pd}_{2} \mathrm{Al}_{3},{ }^{16}$, etc., the residual resistivity $\rho(0)$ rapidly increases with increasing concentration $x$ of the nonmagnetic impurities, and one obtains a crossover from the metallic $\rho(T)$ behavior with a maximum to a monotonic curve, where $\rho(T)$ decreases with increasing $T$. The thermoelectric power $S(T)$ for $\mathrm{CeCu}_{2.05} \mathrm{Si}_{2}$ shows a crossover from one case with a negative minimum, a sign change, and a positive maximum to a behavior with two positive maxima and no sign change when substituting Ce by La. ${ }^{14,15}$ For materials such as $\mathrm{Ce}_{3} \mathrm{Cu}_{x} \mathrm{Pt}_{3-x} \mathrm{Sb}_{4}$, ligand alloying introduces a transition from a Kondo insulator to a dirty metal. ${ }^{12}$

The basic model for a description of the electronic properties of HFSs is the periodic Anderson model ${ }^{17}$ (PAM). In a recent paper, ${ }^{18}$ we studied the PAM within the dynamical meanfield theory ${ }^{19,20}$ (DMFT) and showed that the characteristic transport properties of HFSs can be understood within this framework. Within the DMFT, the lattice model is mapped on an effective single-impurity Anderson model ${ }^{21}$ (SIAM) by a self-consistency condition. We used the numerical renormalization group ${ }^{22,23}$ (NRG) as impurity solver for this effective SIAM. The NRG is a nonperturbative method applicable at very low temperatures which reproduces the correct characteristic low-temperature scale (Kondo temperature). ${ }^{24}$

In the present paper, we extend the DMFT-NRG treatment to the disordered PAM. The disorder is studied within the coherent-potential approximation ${ }^{25,26}$ (CPA). The CPA was originally developed for alloys modeled by noninteracting particles. In the CPA, lattice coherence is restored by introducing an average potential. Janiš and Vollhardt have pointed out that the local nature of the potential allows one to embed the CPA into the DMFT framework ${ }^{27}$ emphasizing that the CPA is the best possible single-site approximation ${ }^{26}$ for disorder. On the other hand, the DMFT can be interpreted as CPA for periodic correlated electron systems as the DMFT reduces to the CPA equations in the absence of 
electron-electron interactions. ${ }^{28}$ This allows for generalizations of the CPA to disordered systems with finite Coulomb repulsion which has been exploited in the context of the Hubbard model. ${ }^{27,29,30,31,32}$

There exists a number of previous works in which the influence of disorder on the electronic properties of HFSs has been studied. ${ }^{33,34,35,36,37,38,39,40,41,42}$ Most of these papers consider the case of disorder by Kondo holes only. The earliest work ${ }^{33,34}$ applied the alloy analog approximation to the PAM as a CPA application. Other treatments ${ }^{36,37,38}$ used the slave-boson mean-field approximation to the PAM, which enabled a straightforward application of the standard CPA. ${ }^{25,26}$ Schlottmann ${ }^{39}$ studied the development of impurity bands within the hybridization gap of Kondo insulators in the low-concentration limit using the iterative pertubation theory (ITP). Mutou ${ }^{41,42}$ and Wermbter et al..$^{40}$ calculated transport properties within the framework of the ITP and secondorder perturbation theory, respectively. Recently, the lowtemperature behavior of paramagnetic Kondo lattices upon random depletion of the local $f$ moments has been investigated by Kaul and Vojta. ${ }^{43}$

We extend the DMFT in order to describe a disordered PAM, based on the CPA for noninteracting systems in the spirit of Janiš and Vollhardt. ${ }^{27}$ In contrast to the standard CPA with static potentials, we use dynamical local potentials which include correlation effects. For reasons of simplicity, we call this extension "CPA", too, and stick to the name "DMFT" for pure systems without disorder. Here, we apply such a generalized CPA to the disordered PAM and calculate the transport quantities $\rho(T)$ and $S(T)$ for disorder on the $f$ site as well as for disorder on the ligand sites. We treat the whole range of impurity concentrations $c \in[0 ; 1]$ for different choices of the PAM parameters, starting with either a metallic HFS or a Kondo insulator for $c=0$. We find that this CPA-NRG treatment of the PAM is able to explain the strong disorder dependence of the transport quantities of HFSs.

A generalization of the CPA for binary alloys to infinitely many local environments has been considered in the context of ligand disorder of $\mathrm{UCu}_{5-x} \mathrm{Pd}_{x}$ by Dobrosavljević and coworkers. ${ }^{44,45,46}$ Those locally different environments yield a distribution of hybridization strengths between the actinide and the conduction band at each site and, as a consequence, a distribution of Kondo scales. By averaging over these different low-energy scales, a non-Fermi-liquid behavior of transport properties was obtained. ${ }^{45,46,47}$

HFSs often exhibit symmetry broken phases such as superconductivity ${ }^{48}$ or antiferromagnetism, ${ }^{2}$ which are certainly not yet properly understood. Such phase transitions at finite temperatures can be qualitatively understood within the PAM, ${ }^{49,50,51,52,53,54,55,56,57,58}$ but the complete phase diagram of the model in three dimensions has not yet been determined. A vanishing transition temperature as a function of an external control parameter such as magnetic field, pressure, or doping defines the quantum critical point (QCP), at which the transition is governed by quantum rather than thermal fluctuations. This sparked a lot of experimental ${ }^{1}$ as well as theoretical ${ }^{59,60,61}$ interest since it is believed that these fluctuations strongly affect the transport properties at a finite tem- perature in a cone close to the QCP. ${ }^{60,61}$ A proper microscopic description of such QCP requires the treatment of one- and two-particle properties on equal footing, which is beyond the scope of the present investigation and must be left for future research. Therefore, we restrict ourselves to the influence of disorder on transport properties in the paramagnetic phase of the model.

The paper is organized as follows: In Sec. II, we introduce the model and the notations as well as our calculation methods, the DMFT, the CPA, and the NRG as impurity solver. Section III is devoted to the special case of Kondo holes: in Sec. III A, simplifications of the CPA equations are considered, and in Sec. III B, we discuss the temperature-dependent spectral functions and self-energy of the PAM. These singleparticle properties determine the transport properties via the Kubo formulas introduced in Sec. IV. We explicitly state equations for the resistivity $\rho$ and the thermoelectric power $S$. In Sec. V, results on the temperature dependence of $\rho(T)$ and $S(T)$ are reported, both for disorder on the $f$ site and disorder on the ligand sites. We conclude with a summary and outlook in Sec. VI.

\section{THEORY}

The essential features of heavy-fermion compounds are based on the interplay of localized, strongly correlated $f$ electrons with broad conduction bands. At high temperature, the weakly coupled $f$ electrons cause mainly incoherent and, with decreasing temperature, logarithmically growing spinflip scattering for the conduction electrons. Below a characteristic temperature scale, a crossover to a coherent lowtemperature phase is observed. The $f$ electrons contribute significantly to the formation of heavy quasiparticles, while their moments are dynamically screened. The PAM takes these ingredients into account, comprising of spin degenerate conduction electrons, a lattice of correlated localized $f$ electrons, and a hybridization (cf. Sec. II A).

In this work, we investigate the influence of disorder in HFSs. In general, alloying with different ingredients $A, B$, $C, \ldots$, with concentrations $c_{A}, c_{B}, c_{C}, \ldots$, respectively, destroys the lattice periodicity. Here, we consider only substitutional alloys of the type $A_{c} B_{1-c}$ with concentrations $c_{A}=c$ and $c_{B}=1-c$.

\section{A. Model}

This section is divided into two parts: In the first part, we will introduce the model for periodic systems, which is extended to binary alloys in the second part. 


\section{No disorder.}

The Hamiltonian of the simplest version of the PAM is given by ${ }^{17}$

$$
\begin{aligned}
\hat{H}= & \sum_{\vec{k} \sigma} \varepsilon_{\vec{k} \sigma} c_{\vec{k} \sigma}^{\dagger} c_{\vec{k} \sigma}+\frac{U}{2} \sum_{i \sigma} \hat{n}_{i \sigma}^{f} \hat{n}_{i-\sigma}^{f} \\
& +\sum_{i \sigma}\left(\begin{array}{ll}
f_{i \sigma}^{\dagger} & c_{i \sigma}^{\dagger}
\end{array}\right) \mathscr{V}_{\sigma}\left(\begin{array}{c}
f_{i \sigma} \\
c_{i \sigma}
\end{array}\right) \text { with } \mathscr{V}_{\sigma}=\left(\begin{array}{cc}
\varepsilon_{f \sigma} & V_{\sigma} \\
V_{\sigma} & \varepsilon_{c \sigma}
\end{array}\right) .
\end{aligned}
$$

Here, $c_{\vec{k} \sigma}\left(c_{\vec{k} \sigma}^{\dagger}\right)$ destroys (creates) a conduction electron with spin $\sigma$, momentum $\vec{k}$, and energy $\varepsilon_{\vec{k} \sigma}+\varepsilon_{c \sigma}$, where $\varepsilon_{c \sigma}$ indicates the band center. The energy $\varepsilon_{f \sigma}$ denotes the spindependent single-particle $f$-level energy at lattice site $i, \hat{n}_{i \sigma}^{f}=$ $f_{i \sigma}^{\dagger} f_{i \sigma}$ is the $f$-electron occupation operator (per site and spin), $f_{i \sigma}\left(f_{i \sigma}^{\dagger}\right)$ destroys (creates) an $f$ electron with spin $\sigma$ at site $i$, and $U$ denotes the on-site Coulomb repulsion between two $f$ electrons on the same site $i$. The uncorrelated conduction electrons hybridize locally with the $f$ electrons via the matrix element $V_{\sigma}$.

Even though only a single effective $f$ level is considered, this model is quite general. It describes any heavyfermion system with odd ground-state filling of the $f$ shell, for which in a strong crystal field environment the degenerate Hund's rule ground state may be reduced to an effective spindegenerate Kramers doublet. In addition, charge fluctuations to even $f$ fillings leave the $f$ shell in crystal field singlets. The Hamiltonian contains four energy scales. The interplay between $\varepsilon_{f \sigma}$ and $U$ controls the average $f$ filling as well as the local moment formation for large $U$ and negative $\varepsilon_{f \sigma}$. The Anderson width $\Gamma_{0}=V^{2} \pi \rho_{0}(0)$ determines the charge fluctuation scale of the $f$ electrons, with $\rho_{0}(0)$ being the density of states of the noninteracting conduction band of width $D$ at its band center.

The total filling per site, $n_{\mathrm{tot}}=\sum_{\sigma}\left(\left\langle\hat{n}_{i \sigma}^{c}\right\rangle+\left\langle\hat{n}_{i \sigma}^{f}\right\rangle\right)$, is kept constant by a temperature-dependent chemical potential $\mu(T)$. We absorb the energy shifts into the band center $\varepsilon_{c \sigma}$ of the conduction band, as well as the $f$ level $\varepsilon_{f \sigma}$. For $n_{\text {tot }}=2$ and $U=0$, the uncorrelated system is an insulator at $T=0$, since the lower of the two hybridized bands is completely filled. According to Luttinger's theorem, a finite $U$ of arbitrary strength does not change the Fermi volume, which includes the full first Brillouin zone. As long as the ground state does not change the symmetry due to a phase transition, the system remains an insulator at arbitrarily large Coulomb repulsion. Therefore, the nonmetallic ground state of Kondo insulators is not correlation induced, but it is already present for the noninteracting system and is a consequence of Luttinger's theorem. For nonintegral values of $n_{\text {tot }}$, the paramagnetic phase of the system must be metallic.

\section{Binary alloy.}

The simplest version (1) of the PAM can be extended to describe two subsystems by replacing the matrix $\mathscr{V}_{\sigma}$ by a random potential $\mathscr{V}_{i \sigma}$. At each lattice site $i$, two different values are possible with a probability $c$ that is given by the relative concentration of subsystem $A$ :

$$
\mathscr{V}_{i \sigma}= \begin{cases}\mathscr{V}_{\sigma}^{A} & \text { with probability } c \\ \mathscr{V}_{\sigma}^{B} & \text { with probability } 1-c\end{cases}
$$

In the case of no doping (i.e., $\mathscr{V}_{\sigma}^{A}=\mathscr{V}_{\sigma}^{B}$ or $c=0$ or $c=1$ ), the extended model reduces to Eq. (1).

While Eq. (1) includes possible Zeeman splitting of the energies in an external magnetic field $H$, we set $H=0$ throughout the remainder of the paper and treat all properties as spin degenerate. In particular, we drop the spin index $\sigma$ from now on for the sake of simplicity.

\section{B. Dynamical mean-field theory}

Setting aside exact solutions in one dimension ${ }^{52}$ using the Bethe ansatz for the Kondo lattice model, to our knowledge no exact analytical solution has been found for the model (1) with finite $U$. Therefore, one has to rely on suitable approximations for the PAM. An obvious first approximation is the assumption of a purely local, site-diagonal (i.e., $\vec{k}$ independent) self-energy, which for the PAM is even better justified than for other lattice models of correlated electron systems, as the first corrections are at least of order $V^{6}$. Within a local self-energy approximation, the complicated lattice model can be mapped on an effective SIAM. ${ }^{21}$ Such a mapping was first used about 20 years ago in connection with the applications of the noncrossing approximation ${ }^{62,63,64,65}$ (NCA) to the PAM. ${ }^{66,67,68,69,70}$ The effective site is viewed as a correlated atomic problem within a time-dependent (or energydependent) external field ${ }^{66,69,70,71}$ which is determined selfconsistently, thus accounting for the feedback of the electron propagation through the lattice. ${ }^{66,67,68}$ Using the scaling of the tight-binding hopping parameter in large dimensions by Metzner and Vollhardt, ${ }^{72}$ Müller-Hartmann ${ }^{73}$ has proven that the local self-energy approximation becomes exact in the limit of infinite spatial dimensions. Therefore, the self-consistency condition of the DMFT, ${ }^{19,20,66,74,75}$ which neglects spatial fluctuations in the single-particle self-energy, becomes exact in the limit $d \rightarrow \infty$. Within weak-coupling $U$-perturbation theory, it could be shown ${ }^{76,77}$ that a local, $\vec{k}$-independent selfenergy is a good approximation for realistic dimension $d=3$ as corrections due to intersite contributions to the self-energy are negligibly small. As a consequence, phase transitions remain mean-field-like in DMFT since $\vec{k}$-dependent fluctuations are not included in a local approximation. ${ }^{20,57,58}$

The following exact relations for the conduction electron Green function $G_{c}(\vec{k}, z)$ and the $f$-electron Green function $G_{f}(\vec{k}, z)$ can be obtained for the PAM (1):

$$
\begin{aligned}
G(\vec{k}, z) & =\left[\begin{array}{cc}
G_{f f}(\vec{k}, z) & G_{f c}(\vec{k}, z) \\
G_{c f}(\vec{k}, z) & G_{c c}(\vec{k}, z)
\end{array}\right] \\
& =\left\{\left(\begin{array}{cc}
z & 0 \\
0 & z-\varepsilon_{\vec{k}}
\end{array}\right)-\left[\begin{array}{cc}
\varepsilon_{f}+\Sigma_{f}(\vec{k}, z) & V \\
V & \varepsilon_{c}
\end{array}\right]\right\}^{-1},
\end{aligned}
$$


where $z$ is any complex energy off the real axis. Within a local approximation such as the DMFT, the $\vec{k}$-dependent $f$ electron self-energy $\Sigma_{f}(\vec{k}, z)$ is replaced by a $\vec{k}$-independent $\Sigma_{f}(z)$. From Eq. (3), one defines a self-energy of the conduction electrons via

$$
\Sigma_{c}(z)=\frac{V^{2}}{z-\varepsilon_{f}-\Sigma_{f}(z)}
$$

which can include a simple $\vec{k}$ dependence through the hybridization matrix elements $V^{2}$, here taken as a constant. For such a local self-energy $\Sigma_{c}$, the site-diagonal conductionelectron Green function $G_{c c}$ can be written as a Hilbert transformation

$$
G_{c c}(z)=\frac{1}{N} \sum_{\vec{k}} G_{c c}(\vec{k}, z)=D\left[z-\varepsilon_{c}-\Sigma_{c}(z)\right],
$$

defined for arbitrary complex argument $z$ as

$$
D(z)=\int_{-\infty}^{\infty} d \varepsilon \frac{\rho_{0}(\varepsilon)}{z-\varepsilon}
$$

where $\rho_{0}(\omega)$ is the density of states of the noninteracting conduction electrons.

The DMFT self-consistency condition states that the sitediagonal matrix element of the $f$-electron Green function of the PAM must be equal to $G_{f f}^{\text {loc }}(z)$ of an effective site problem

$$
\begin{aligned}
& G_{f f}(z)=\frac{1}{N} \sum_{\vec{k}} G_{f f}(\vec{k}, z)=G_{f f}^{\mathrm{loc}}(z), \\
& G_{f f}^{\mathrm{loc}}(z)=\frac{1}{z-\varepsilon_{f}-\Delta(z)-\Sigma_{f}(z)},
\end{aligned}
$$

with the same local $f$-electron self-energy $\Sigma_{f}(z)$ for the lattice and the effective site. This defines the self-consistency condition for the functions $\Sigma_{f}(z)$ and $\Delta(z)$.

We can put the site-diagonal Green functions into a Green function matrix

$$
G(z)=\left[\begin{array}{ll}
G_{f f}(z) & G_{f c}(z) \\
G_{c f}(z) & G_{c c}(z)
\end{array}\right]
$$

but it is sufficient to know one of the components because they are connected to each other:

$$
G_{f c}(z)=G_{c f}(z)=\frac{\Sigma_{c}(z)}{V} G_{c c}(z)=\frac{\Delta(z)}{V} G_{f f}(z) .
$$

Furthermore, the self-consistency condition (7) extends to the full matrix, $G(z)=G^{\mathrm{loc}}(z)$. It is possible to formulate the DMFT equation for the conduction-electron Green function instead of the $f$-electron Green function:

$$
\Delta(z)=z-\varepsilon_{f}-\Sigma_{f}(z)-G_{f f}(z)^{-1}=\frac{V^{2}}{\Sigma_{c}(z)+G_{c c}(z)^{-1}} .
$$

This condition can also be written in the following form:

$$
\left(\begin{array}{cc}
z & 0 \\
0 & z
\end{array}\right)-\left[\begin{array}{cc}
\varepsilon_{f}+\Sigma_{f}(z) & V \\
V & \varepsilon_{c}
\end{array}\right]-G^{-1}(z)=\left[\begin{array}{cc}
0 & 0 \\
0 & z-\varepsilon_{c}-\frac{V^{2}}{\Delta(z)}
\end{array}\right] .
$$

Given the Green functions $G_{f f}(z)$ and $G_{c c}(z)$, their spectral functions

$$
\begin{gathered}
\rho_{f}(\omega)=\operatorname{Im} G_{f f}\left(\omega-i 0^{+}\right) / \pi, \\
\rho_{c}(\omega)=\operatorname{Im} G_{c c}\left(\omega-i 0^{+}\right) / \pi
\end{gathered}
$$

determine the local occupation numbers

$$
n_{f / c}=\sum_{\sigma} \int_{-\infty}^{\infty} d \omega f(\omega-\mu) \rho_{f / c}(\omega)
$$

where the spin sum contributes only a factor of 2 in the absence of a magnetic field and $f(\omega)$ denotes the Fermi function. Then, the total filling per site is given by $n_{\text {tot }}=n_{f}+n_{c}$. Particle-hole symmetry is reached at $n_{\text {tot }}=2$ and $\varepsilon_{f}-\varepsilon_{c}=$ $-U / 2$ for a symmetric $\rho_{0}(\omega)$. As a matter of convenience, we will perform an integral transformation such that $\mu$ is absorbed into $\varepsilon_{f}$ and the band center $\varepsilon_{c}$; all energies will be measured with respect to $\mu$. For a given lattice filling $n_{\text {tot }}$, we have to adjust $\mu$ in addition to fulfill Eq. (7).

Before we discuss the solution of the effective site, let us briefly comment on the implications of the analytical form of the conduction-electron self-energy (4). For a Fermi liquid at $T \rightarrow 0$, the imaginary part of $\Sigma_{f}$ vanishes quadratically close to the chemical potential, i.e., $\operatorname{Im} \Sigma_{f}\left(\omega-i 0^{+}\right) \propto \omega^{2}$. At particle-hole symmetry, $\Sigma_{c}(z)$ diverges as $1 / z$, leading to an insulator. Away from particle-hole symmetry, the denominator remains finite and the imaginary part also must have Fermi-liquid properties $\operatorname{Im} \Sigma_{c}\left(\omega-i 0^{+}\right) \propto \operatorname{Im} \Sigma_{f}\left(\omega-i 0^{+}\right) \propto$ $\omega^{2}$. The real part is very large, and therefore, the spectral function $\rho_{c}(\omega)$ as well as $\rho_{f}(\omega)$ sample the high-energy band edges of $\rho_{0}(\omega)$, yielding a hybridization gap. These analytic properties must be fulfilled by any approximate solution of the DMFT self-consistency condition (7) consistent with Fermiliquid theory.

\section{Coherent-potential approximation}

The DMFT is only applicable to periodic systems. When we consider alloys or doped systems where the lattice periodicity is destroyed, then the DMFT has to be extended. There are several possible approximations ${ }^{26}$ for disordered alloys, e.g., the virtual-crystal method, the average t-matrix approximation, ${ }^{78}$ or the CPA. ${ }^{25,26,32,79}$ The CPA is the best single-site approximation ${ }^{26,28}$ and can be seen as an extension of the DMFT for two subsystems. If there is effectively only one subsystem (as in the case of $\mathscr{V}_{A}=\mathscr{V}_{B}$ or $c=0$ or $c=1$ ), the CPA reduces to the DMFT.

The standard CPA self-consistency condition for noncorrelated systems is given by

$$
\begin{aligned}
c & \left\{\mathbb{1}-\left[\mathscr{V}_{A}-\Sigma^{\mathrm{CPA}}\right] \mathscr{G}\right\}^{-1}\left[\mathscr{V}_{A}-\Sigma^{\mathrm{CPA}}\right] \\
& +(1-c)\left\{\mathbb{1}-\left[\mathscr{V}_{B}-\Sigma^{\mathrm{CPA}}\right] \mathscr{G}\right\}^{-1}\left[\mathscr{V}_{B}-\Sigma^{\mathrm{CPA}}\right]=0
\end{aligned}
$$


where $\Sigma^{\mathrm{CPA}}(z)$ is the self-energy (matrix) of the configurationally averaged Green function (matrix)

$$
\begin{aligned}
\mathscr{G}(z) & =\left[\begin{array}{cc}
\mathscr{G}_{f f}(z) & \mathscr{G}_{f c}(z) \\
\mathscr{G}_{c f}(z) & \mathscr{G}_{c c}(z)
\end{array}\right] \\
& =\frac{1}{N} \sum_{\vec{k}}\left[\left(\begin{array}{cc}
z & 0 \\
0 & z-\varepsilon_{\vec{k}}
\end{array}\right)-\Sigma^{\mathrm{CPA}}(z)\right]^{-1}
\end{aligned}
$$

[as in Eq. (5), the CPA Green function matrix can also be written as a Hilbert transformation by replacing the $\vec{k}$ sum with the integral $D]$.

To include correlation effects in Eq. (14), the potential matrix $\mathscr{V}_{A / B}$ of subsystems $A$ and $B$, respectively, has to be replaced by the sum

$$
\mathscr{V}_{A / B} \leadsto \mathscr{V}_{A / B}+\left[\begin{array}{cc}
\Sigma_{f}^{A / B}(z) & 0 \\
0 & 0
\end{array}\right],
$$

where $\Sigma_{f}^{A / B}(z)$ is the local $f$-electron self-energy of subsystems $A$ and $B$, respectively. Equivalent to Eq. (14) with correlation is the following CPA equation:

$$
\mathscr{G}(z)=c G^{A}(z)+(1-c) G^{B}(z),
$$

where $G^{A}$ and $G^{B}$ are the local Green function matrices (9) of effective site problems for the two subsystems $A$ and $B$, given by the effective media $\Delta_{A}(z)$ and $\Delta_{B}(z)$, respectively:

$$
\begin{gathered}
G_{f f}^{A / B}(z)=\left[z-\varepsilon_{f}^{A / B}-\Delta_{A / B}(z)-\Sigma_{f}^{A / B}(z)\right]^{-1}, \\
G_{f c}^{A / B}(z)=\frac{\Sigma_{c}^{A / B}(z)}{V_{A / B}} G_{c c}^{A / B}(z)=\frac{\Delta_{A / B}(z)}{V_{A / B}} G_{f f}^{A / B}(z) .
\end{gathered}
$$

Note that the determinant of $\mathscr{G}$ [Eq. (15)] can be written in the following simple form:

$$
\operatorname{det} \mathscr{G}(z)=\frac{\mathscr{G}_{c c}(z)}{z-\Sigma_{f f}^{\mathrm{CPA}}} .
$$

Using this expression, it can be shown by direct calculation that

$$
\left(\begin{array}{cc}
z & 0 \\
0 & z
\end{array}\right)-\Sigma^{\mathrm{CPA}}(z)-\mathscr{G}^{-1}(z)=\left[\begin{array}{cc}
0 & 0 \\
0 & \Gamma(z)
\end{array}\right]
$$

has got one component only. Therefore, we obtain the same structure as in the case of the DMFT, Eq. (11). This leads to a DMFT-like self-consistency for the effective medium by setting

$$
\Delta_{A / B}(z):=\frac{V_{A / B}^{2}}{z-\varepsilon_{C}^{A / B}-\Gamma(z)} .
$$

This ensures that for pure systems $(c=0$ or $c=1)$ the DMFT limit as given by Eq. (11) is recovered.

As in Eqs. (12) and (13), the CPA spectral functions

$$
\begin{aligned}
& \rho_{f}^{\mathrm{CPA}}(\omega)=\operatorname{Im} \mathscr{G}_{f f}\left(\omega-i 0^{+}\right) / \pi, \\
& \rho_{c}^{\mathrm{CPA}}(\omega)=\operatorname{Im} \mathscr{G}_{c c}\left(\omega-i 0^{+}\right) / \pi
\end{aligned}
$$

determine the occupation numbers

$$
n_{f / c}=\sum_{\sigma} \int_{-\infty}^{\infty} d \omega f(\omega-\mu) \rho_{f / c}^{\mathrm{CPA}}(\omega) .
$$

In analogy to Eq. (4), we define

$$
\Sigma_{c}^{\mathrm{CPA}}(z):=\frac{\Sigma_{f c}^{\mathrm{CPA}}(z) \Sigma_{c f}^{\mathrm{CPA}}(z)}{z-\Sigma_{f f}^{\mathrm{CPA}}(z)} .
$$

The CPA self-consistency cycle consists of two parts: (I) After solving the two impurity subsystems $A$ and $B$ for the given effective media, the CPA self-energy matrix is determined by Eqs. (17) and (20). (II) A new estimate for the CPA Green function matrix is obtained by Eq. (15). Both matrices are required to calculate $\Gamma(z)$-and thus two new effective media—by Eq. (20). The cycle is completed by shifting $\varepsilon_{f}^{A / B}$ and $\varepsilon_{c}^{A / B}$ such that $N[G]=n_{\text {tot }}$ and $\mu=0$.

Although the CPA includes the DMFT as end points for the concentrated systems $(c=0$ and $c=1)$, conceptual differences in disordered systems arise from the occurrence of the additional quantities. In the CPA, we must distinguish between the local matrix $\mathscr{V}_{A / B}$, which describes the properties of a site of type $A / B$ and differs at different sites, and the configuration averaged $k$-independent lattice self-energy $\Sigma^{\mathrm{CPA}}(z)$, which is equal for all lattice sites. Moreover, the configurationally averaged CPA Green function restores translational invariance in the lattice and is not equal to either local Green function $G^{A / B}(z)$. Even though the CPA yields only one configurationally averaged dynamical field $\Gamma(z)$, the local dynamics of each site is determined by different media $\Delta_{A / B}(z)$. Only for $c=0$ and $c=1$, the CPA equation (17) is trivially fulfilled and $\Sigma^{\mathrm{CPA}}(z)$ coincides with the local self-energy as well as the CPA Green function $\mathscr{G}(z)$ coincides with the one of the effective site.

\section{Impurity solver}

The local $f$-electron self-energy $\Sigma_{f}^{A / B}$ entering the CPA equation (17) via the local Green function matrix $G^{A / B}$ [Eq. (18)] is obtained for an effective site defined by the local Hamiltonian ${ }^{18,19,20,67,74,75} \hat{H}_{\text {eff }}^{A / B}$ for subsystems $A$ and $B$, respectively,

$$
\begin{aligned}
\hat{H}_{\mathrm{eff}}^{A / B}= & \sum_{\sigma}\left(\varepsilon_{f \sigma}^{A / B}-\mu\right) f_{\sigma}^{\dagger} f_{\sigma}+U \hat{n}_{\uparrow} \hat{n}_{\downarrow}+\sum_{\sigma} \int d \varepsilon(\varepsilon-\mu) d_{\sigma \varepsilon}^{\dagger} d_{\sigma \varepsilon} \\
& +\sum_{\sigma} \int d \varepsilon V_{A / B} \sqrt{\rho_{\mathrm{eff}}^{A / B}(\varepsilon)}\left(d_{\sigma \varepsilon}^{\dagger} f_{\sigma}+f_{\sigma}^{\dagger} d_{\sigma \varepsilon}\right)
\end{aligned}
$$

The coupling of the $f$ electron to a fictitious bath of "conduction electrons" created by $d_{\sigma \varepsilon}^{\dagger}$ is described by an energydependent hybridization function

$$
\pi V_{A / B}^{2} \rho_{\mathrm{eff}}^{A / B}(\varepsilon)=\operatorname{Im} \Delta_{A / B}\left(\varepsilon-i 0^{+}\right) .
$$


Using Eq. (21), we can write the density of states of the fictitious bath as

$$
\rho_{\mathrm{eff}}^{A / B}(\varepsilon)=\frac{1}{\pi} \operatorname{Im}\left[\varepsilon-i 0^{+}-\varepsilon_{c}^{A / B}-\Gamma\left(\varepsilon-i 0^{+}\right)\right]^{-1} .
$$

We accurately solve the Hamiltonian (25) using Wilson's NRG approach. ${ }^{22,23}$ The key ingredient in the NRG is a logarithmic discretization of the continuous bath, controlled by the parameter ${ }^{22} \Lambda>1$. The Hamiltonian is mapped onto a semi-infinite chain, where the $N$ th link represents an exponentially decreasing energy scale $D_{N} \sim \Lambda^{-N / 2}$. Using this hierarchy of scales, the sequence of finite-size Hamiltonians $\mathscr{H}_{N}$ for the $N$-site chain is solved iteratively, discarding the highenergy states at each step to maintain a manageable number of states. The reduced basis set of $\mathscr{H}_{N}$ thus obtained is expected to faithfully describe the spectrum of the full Hamiltonian on the scale of $D_{N}$, corresponding ${ }^{22}$ to a temperature $T_{N} \sim D_{N}$ from which all thermodynamic expectation values are calculated. The energy-dependent hybridization function $\Delta(z)$ determines the coefficients of the semi-infinite chain. ${ }^{18,80}$ For further details, we refer to the recent NRG review by Bulla $e t$ $a l .{ }^{81}$

The finite-temperature NRG spectral functions are calculated using the recently developed algorithm from Refs. 82 and 83. The usage of a complete basis set of the Wilson chain originally derived for real-time dynamics of quantum impurites out of equilibrium ${ }^{84,85}$ ensures that the spectral sum rule is exactly fulfilled and that the NRG occupancy is accurately reproduced for arbitrary values of $\Lambda$ and number of retained states $N_{s}$. The NRG spectrum is broadened in the usual way ${ }^{81,86,87}$ by a Gaussian at higher frequency and a Lorentzian for low-frequency excitations $|E|$

$$
\delta(\omega-E) \leadsto \begin{cases}\frac{e^{-b^{2} / 4}}{\sqrt{\pi} b|E|} e^{-[\log (\omega / E) / b]^{2}} & \text { for }|E| \geq L_{w} T \\ \frac{1}{\pi} \frac{L_{T} T}{(\omega-E)^{2}+\left(L_{T} T\right)^{2}} & \text { for }|E|<L_{w} T .\end{cases}
$$

As long as not stated otherwise, we use

$$
b=0.6, \quad L_{T}=1, \quad L_{w}=\sqrt{\pi} L_{T} \exp \left(-b^{2} / 4\right) / b .
$$

The local $f$-electron self-energy $\Sigma_{f}^{A / B}$ is determined by the exact ratio

$$
\Sigma_{\sigma}^{f}(z)=U \frac{M_{\sigma}^{\mathrm{NRG}}(z)}{F_{\sigma}^{\mathrm{NRG}}(z)}
$$

derived via equation of motion technique, ${ }^{88}$ where the correlation functions $M_{\sigma}^{\mathrm{NRG}}(z)=\left\langle\left\langle f_{\sigma} f_{-\sigma}^{\dagger} f_{-\sigma} \mid f_{\sigma}^{\dagger}\right\rangle\right\rangle(z)$ and $F_{\sigma}^{\mathrm{NRG}}(z)=\left\langle\left\langle f_{\sigma} \mid f_{\sigma}^{\dagger}\right\rangle\right\rangle(z)$ have been obtained from NRG spectral functions.

We use a Gaussian model density of states

$$
\rho_{0}(\varepsilon)=\frac{\exp \left[-\left(\varepsilon / t^{*}\right)^{2} / 2\right]}{t^{*} \sqrt{2 \pi}}
$$

for the unperturbed conduction-electron system. In the following, we set $\sqrt{2} t^{*}=10 \Gamma_{0}$ and measure energies in units of $\Gamma_{0}=\pi V_{0}^{2} \rho_{0}(0)$ which defines a reference hybridization $V_{0}$ by
$V_{0}^{2}=2 t^{*} \Gamma_{0} / \sqrt{2 \pi}=10 \Gamma_{0}^{2} / \sqrt{\pi} \approx 5.64 \Gamma_{0}^{2}$. In order to make contact to the experimentally relevant parameter regime, we assume $\Gamma_{0}=100 \mathrm{meV}$, which translates into a temperature scale of $\frac{\Gamma_{0}}{k_{B}} \approx 1160 \mathrm{~K}$.

\section{KONDO HOLES}

\section{A. Analytical considerations}

A special case for disorder on the $f$ site is given by introducing Kondo holes, i.e., lattice sites without $f$ electrons. We describe the two subsystems with identical parameters $\left(V:=V_{A}=V_{B}, \varepsilon_{c}:=\varepsilon_{c}^{A}=\varepsilon_{c}^{B}\right.$, and therefore $\left.\Delta:=\Delta_{A}=\Delta_{B}\right)$ except that the $f$-level energy of system $B$ is shifted toward infinity. Additionally, the total electron density is reduced by one electron per $B$ site to $n_{\text {tot }}=n_{\text {tot }}(c=1)-(1-c)$.

Because there are no $f$ electrons in system $B$, the conduction-electron self-energy $\Sigma_{c}^{B}$ [Eq. (4)] vanishes. By Eqs. (9) and (11), we conclude that the Green function matrix of system $B$ contains only the $c c$ element:

$$
G^{B}(z)=\left(\begin{array}{cc}
0 & 0 \\
0 & \Delta(z) / V^{2}
\end{array}\right) .
$$

As a consequence, the configurationally averaged $f$-electron Green function of Eq. (17) is given by $\mathscr{G}_{f f}(z)=c G_{f f}^{A}(z)$, vanishing for $c \rightarrow 0$, and the configurationally averaged bandelectron Green function $\mathscr{G}_{c c}(z)$ interpolates between $G_{c c}^{A}(z)$ and the free medium with band center at $\varepsilon_{c}$.

By inserting Eq. (17) into Eq. (20), we conclude that in the case of Kondo holes the CPA self-energy matrix reduces to a scalar self-energy

$$
\Sigma^{\mathrm{CPA}}(z)=\left[\begin{array}{cc}
\Sigma_{f f}^{\mathrm{CPA}}(z) & V \\
V & \varepsilon_{c}
\end{array}\right]
$$

with

$$
\begin{aligned}
\Sigma_{f f}^{\mathrm{CPA}}(z) & =z-\left[\mathscr{G}^{-1}(z)\right]_{f f}=z-\Delta_{A}(z)-\frac{1}{c G_{f f}^{A}(z)} \\
& =\left(1-\frac{1}{c}\right)[z-\Delta(z)]+\frac{1}{c}\left[e_{f}^{A}+\Sigma_{f}^{A}(z)\right] .
\end{aligned}
$$

There are two contributions to the imaginary part of $\Sigma_{f f}^{\mathrm{CPA}}$ : a term $\frac{1}{c} \operatorname{Im} \Sigma_{f}^{A}$ and a term $\frac{1-c}{c} \operatorname{Im} \Delta$. If $\Sigma_{f}^{A}$ remains Fermiliquid-like for $\omega, T \rightarrow 0$, a finite life time is introduced by the additional $\frac{1-c}{c} \operatorname{Im} \Delta(0)$ for $c<1$. We thus interpolate between the following DMFT limits:

$$
\begin{aligned}
\lim _{c \rightarrow 1}\left[c \Sigma_{f f}^{\mathrm{CPA}}(z)\right] & =\varepsilon_{f}^{A}+\Sigma_{f}^{A}(z), \\
\lim _{c \rightarrow 0}\left[c \Sigma_{f f}^{\mathrm{CPA}}(z)\right] & =-\frac{1}{G_{f f}^{A}(z)}, \\
\lim _{c \rightarrow 1}\left[\frac{1}{c} \Sigma_{c}^{\mathrm{CPA}}(z)\right] & =\Sigma_{c}^{A}(z), \\
\lim _{c \rightarrow 0}\left[\frac{1}{c} \Sigma_{c}^{\mathrm{CPA}}(z)\right] & =V^{2} G_{f f}^{A}(z) .
\end{aligned}
$$


Because the CPA mixes two subsystems, it is not clear how to define a low-temperature scale for the complete system. Each effective site has its own low-temperature scale that characterizes the local dynamics. In the case of Kondo holes, we are in a better situation. As stated above, the configurationally averaged $f$-electron Green function is directly connected to the $f$-electron Green function of subsystem $A$ : $\mathscr{G}_{f f}(z)=c G_{f f}^{A}(z)$, there are no $f$ electrons in system $B$ and, hence, no special low-temperature effects. Then we can take the low-temperature scale of subsystem $A$ to define a lowtemperature scale $T_{\text {low }}$ for the complete system which is only defined up to a constant factor. We will use the renormalization of the Anderson width by the quasiparticle spectral weight

$$
T_{0}=\Gamma_{0}\left[1-\left.\frac{\partial \operatorname{Re} \Sigma_{f}^{A}(\omega)}{\partial \omega}\right|_{\omega, T \rightarrow 0}\right]^{-1}
$$

as our choice of such a low-temperature scale $T_{0} \propto T_{\text {low }}$ for our numerical analysis. ${ }^{89}$ It is related to the mass enhancement $m^{*} / m=\Gamma_{0} / T_{0}$.

\section{B. Spectral properties}

In this section, we take a look at the spectral properties of a system with Kondo holes.

The typical structure of the $f$-electron spectral function $\frac{1}{c} \rho_{f}^{\mathrm{CPA}}(\omega)=\rho_{f}^{A}(\omega)$ is shown in Fig. 1. A pronounced peak structure dominates the low-energy part of the spectrum in the vicinity of the chemical potential. In addition, we observe two shallow high-energy peaks: one at $\varepsilon_{f}$ below $\mu$, and one at $\varepsilon_{f}+U$, which corresponds to double occupancy of the $f$ levels. It is always correctly positioned by the NRG, independent of the value of $U$, but with a linewidth too large due to the NRG broadening procedure of Eq. (27) (see Refs. 87 and 88 for details).

The dependence on the concentration $c$ is small, but the peak width is enlarged with decreasing $c$. This indicates an increasing low-temperature scale; indeed, the scale given by Eq. (33) and shown in the inset of Fig. 1(b) increases with decreasing $c$.

The band-electron spectral function is depicted in Fig. 2. Equivalent to the vanishing of $f$ electrons from the system with decreasing $c$ is the disappearing of the gap structure and the crossover to a free medium of Gaussian shape as discussed in the previous section.

The behavior of the spectral functions is reflected in the conduction-electron self-energy $\frac{1}{c} \Sigma_{c}^{\mathrm{CPA}}(z)$ (cf. Fig. 3). At low temperatures and $c=1$, we observe a characteristic $\omega^{2}$ dependence at the chemical potential $\mu=0$, indicating a Fermi liquid. At $c=0$, the self-energy is proportional to $G_{f f}^{A}$. The imaginary part of the conduction-electron self-energy at a temperature of $274 \mathrm{~K}$ can be seen in Fig. 3(b). Additionally, the derivative of the Fermi function, $f^{\prime}$, is shown. The product of $f^{\prime}$ and $\operatorname{Im} \Sigma_{c}^{\mathrm{CPA}}$ enters the transport integrals in Sec. IV. That is, only the part of $\operatorname{Im} \Sigma_{c}^{\mathrm{CPA}}$ around $\omega=0$ is relevant for
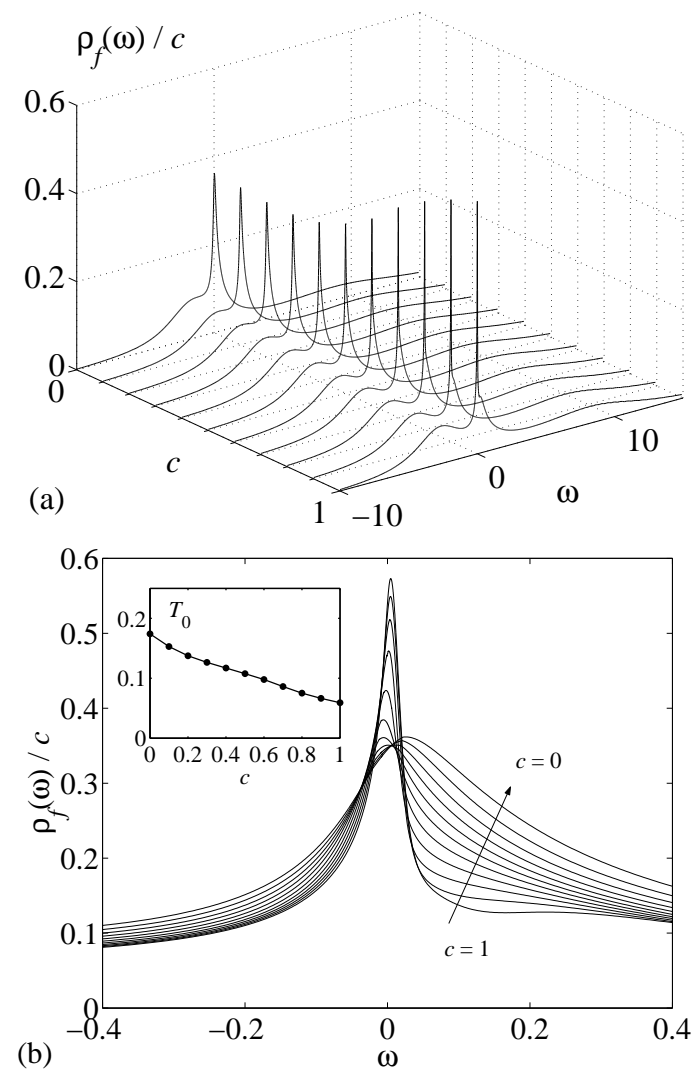

FIG. 1: Spectral density $\rho_{f}(\omega)$ for different concentrations of system $A, c \in\left\{10^{-4}, 0.1,0.2,0.3,0.4,0.5,0.6,0.7,0.8,0.9,1\right\}$, calculated with CPA-NRG for $V_{A}=V_{B}=V_{0}, U / \Gamma_{0}=10, \varepsilon_{f}^{A}-\varepsilon_{c}^{A}=$ $-U / 2, \varepsilon_{f}^{B}=\infty$, chemical potential $\mu=0$, and a filling $n_{\text {tot }}=$ $1.6-(1-c)$ at a small temperature $0.2 \mathrm{~K}$. (a) Overview of the generic three-peak structure, (b) Peak at $\omega=0$ in more detail with corresponding temperature scale $T_{0}$ [Eq. (33)] in the inset. NRG parameters: number of retained NRG states, $N_{s}=800, \Lambda=1.6$, and $\delta / \Gamma_{0}=10^{-3}$.

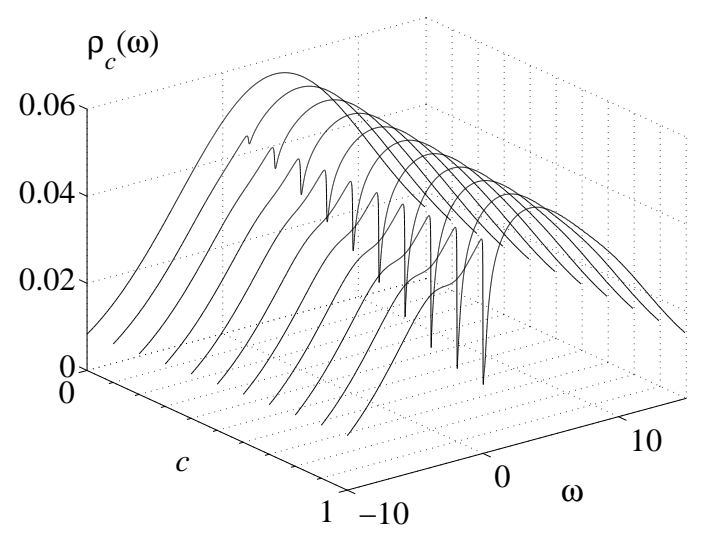

FIG. 2: Spectral density $\rho_{c}(\omega)$, with all parameters as in Fig. 1. With $c \rightarrow 0$, the gap at $\omega=0$ vanishes and we reach a Gaussian curve. 


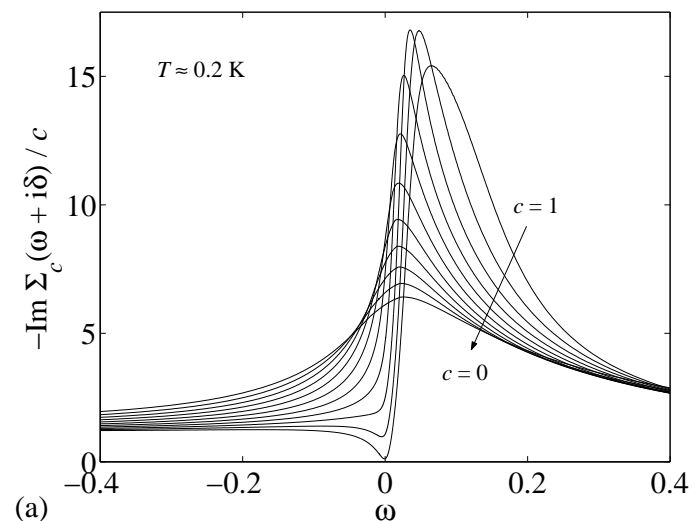

(a)

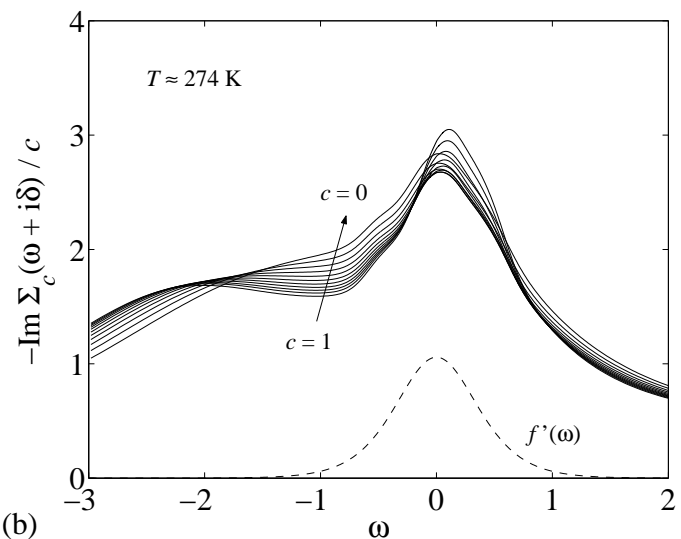

(b)

$\omega$

FIG. 3: Band self-energy $\frac{1}{c} \Sigma_{c}^{\mathrm{CPA}}$ for different concentrations of system $A, c \in\left\{10^{-4}, 0.1,0.2,0.3,0.4,0.5,0.6,0.7,0.8,0.9,1\right\}$, and all parameters as in Fig. 1: (a) at $T \approx 0.2 \mathrm{~K}$, (b) at $T \approx 274 \mathrm{~K}$, with additional derivative of Fermi function, $f^{\prime}$, showing the relevant part of the energy axis.

transport calculations, and in this range, the imaginary part of the conduction-electron self-energy is nearly independent of the concentration $c$.

\section{TRANSPORT THEORY}

To describe the electronic transport within the PAM, we start from the standard relations ${ }^{90}$ for the generalized transport coefficients, according to which the electrical current density $\vec{J}$ and the heat current density $\vec{q}$ depend linearly on the electric field $\vec{E}$ and the temperature gradient $\nabla T$ :

$$
\begin{aligned}
& \vec{J}=L_{11} \vec{E}+L_{12}\left(-\frac{1}{T} \nabla T\right), \\
& \vec{q}=L_{21} \vec{E}+L_{22}\left(-\frac{1}{T} \nabla T\right) .
\end{aligned}
$$

All coefficients are calculated within the linear response approach, starting from similar Kubo formulas. ${ }^{90,91}$ For symmetry reasons, $L_{12}=L_{21}$ must hold.

For example, the real part of the frequency-dependent (optical) conductivity tensor ${ }^{90,92,93} \sigma(\omega)=L_{11}(\omega)$ is related to the current-current correlation function and is written as

$$
\sigma_{\alpha \beta}(\omega)=-\frac{1}{\omega N V_{0}} \operatorname{Im}\left\langle\left\langle j_{\alpha} \mid j_{\beta}^{\dagger}\right\rangle\right\rangle\left(\omega+i 0^{+}\right),
$$

where $V_{0}=a^{3}$ is the volume of the unit cell and $N$ counts the number of lattice sites. It has been shown ${ }^{34}$ that the current operator of the PAM has two contributions: a conductionelectron part and a part proportional to $\nabla V_{\vec{k}}$. The (bare) $f$ electrons do not appear in the current, since they do not disperse. For a $\vec{k}$-independent hybridization, only the conduction electrons carry the electrical and heat currents as follows:

$$
\vec{j}=e \sum_{\vec{k} \sigma} \overrightarrow{v_{\vec{k}}} c_{\vec{k} \sigma}^{\dagger} c_{\vec{k} \sigma}
$$

where $\vec{v}_{\vec{k}}=\frac{1}{\hbar} \nabla_{\vec{k}} \varepsilon_{\vec{k}}$ is the group velocity. Hence, the currentsusceptibility tensor $\left\langle\left\langle\vec{j} \mid \overrightarrow{j^{\dagger}}\right\rangle\right\rangle(z)$ is connected to the particlehole Green function

$$
\left\langle\left\langle\vec{j} \mid \overrightarrow{j^{\dagger}}\right\rangle\right\rangle(z)=e^{2} \sum_{\sigma \sigma^{\prime} \vec{k} \vec{k}^{\prime}} \vec{v}_{\vec{k}} \vec{v}_{\vec{k}^{\prime}}^{T}\left\langle\left\langle c_{\vec{k} \sigma}^{\dagger} c_{\vec{k} \sigma} \mid c_{\vec{k}^{\prime} \sigma^{\prime}}^{\dagger} c_{\overrightarrow{k^{\prime}} \sigma^{\prime}}\right\rangle\right\rangle(z) .
$$

In a cubic crystal, the conductivity is isotropic: $\sigma_{\alpha \beta}(\omega)=$ $\sigma(\omega) \mathbb{1}$. From now on, we will consider only the $x x$ component of the conductivity $\sigma(\omega) \equiv \sigma_{x x}(\omega)$.

In general, the full two-particle Green function $\left\langle\left\langle c_{\vec{k} \sigma}^{\dagger} c_{\vec{k} \sigma} \mid c_{\overrightarrow{k^{\prime}} \sigma^{\prime}}^{\dagger} c_{\vec{k}^{\prime} \sigma^{\prime}}\right\rangle\right\rangle(z)$ involves vertex corrections which reflect residual particle-particle interactions. ${ }^{90}$ However, in the limit $d \rightarrow \infty$, it was shown that current operator vertex corrections vanish. ${ }^{94,95}$ Thus, it is consistent with the DMFT assumption of a $\vec{k}$-independent self-energy that these vertex corrections vanish for any lattice model of correlated electron systems. For the special case of a local approximation for the PAM, this was already shown in Refs. 96, 97, and 78, as for symmetry reasons $\sum_{\vec{k}} \vec{v}_{\vec{k}}\left|V_{\vec{k}}\right|^{2} G_{\vec{k}}(z+\omega) G_{\vec{k}}(z)=0$. The CPA maintains the locality of the self-energy and we can neglect vertex corrections in the case of the CPA, too (see also Ref. 79). All DMFT formulas can be used for the CPA; we only have to substitute the DMFT self-energies with CPA self-energies. In both cases, we obtain

$$
\begin{aligned}
\left\langle\left\langle j_{x} \mid j_{x}^{\dagger}\right\rangle\right\rangle\left(\omega+i 0^{+}\right)= & \frac{2 e^{2}}{\hbar^{2}} \sum_{\vec{k}}\left(\frac{\partial \varepsilon_{\vec{k}}}{\partial k_{x}}\right)^{2} \int_{-\infty}^{\infty} d \omega^{\prime} f\left(\omega^{\prime}\right) \rho_{c}\left(\varepsilon_{\vec{k}}, \omega^{\prime}\right) \\
& \times\left[G_{c c}\left(\vec{k}, \omega^{\prime}+\omega+i 0^{+}\right)\right. \\
& \left.+G_{c c}\left(\vec{k}, \omega^{\prime}-\omega-i 0^{+}\right)\right] .
\end{aligned}
$$

Within the DMFT or the CPA, the lattice one-particle Green function depends only on the (complex) energy $z$ and bare band dispersion $\varepsilon_{\vec{k}}: G_{c c}(\vec{k}, z)=G_{c c}\left(\varepsilon_{\vec{k}}, z\right)$. Then

$$
\frac{1}{N} \sum_{\vec{k}}\left(\frac{\partial \varepsilon_{\vec{k}}}{\partial k_{x}}\right)^{2} A\left(\varepsilon_{\vec{k}}\right)=\int_{-\infty}^{\infty} d \varepsilon \tilde{\rho}_{0}(\varepsilon) A(\varepsilon),
$$

with

$$
\tilde{\rho}_{0}(\varepsilon)=\frac{1}{N} \sum_{\vec{k}}\left(\frac{\partial \varepsilon_{\vec{k}}}{\partial k_{x}}\right)^{2} \delta\left(\varepsilon-\varepsilon_{\vec{k}}\right) .
$$

We use a Gaussian model density of states [Eq. (29)] for the unperturbed conduction-electron system which is appropriate for a $d$-dimensional hypercubic lattice in the limit $d \rightarrow$ 
$\infty,{ }^{72}$ and $\tilde{\rho}_{0}(\varepsilon)$ has been evaluated approximately in large dimensions $^{98}$ as

$$
\tilde{\rho}_{0}(\varepsilon)=\frac{\left(a t^{*}\right)^{2}}{d} \rho_{0}(\varepsilon)+O\left(d^{-2}\right)
$$

on a hypercubic lattice. Then, Eq. (38) can be reduced to a sum of Hilbert transforms, which is defined in Eq. (6).

By taking the limit $\omega \rightarrow 0$, the static conductivity $\sigma=L_{11}$ and the thermoelectric power

$$
S=\frac{1}{T} \frac{L_{12}}{L_{11}}=\frac{k_{B}}{e} \frac{e L_{12}}{k_{B} T L_{11}}
$$

are obtained..$^{90,93}$ The thermoelectric power $S$ is defined as the proportionality constant between an applied temperature gradient and the measured voltage drop in the absence of a current flow. The Peltier coefficient, given by the ratio of heat and electrical current, is related to the thermoelectric power by $\Pi=T S$.

In the limit $\omega \rightarrow 0$, we get for the generalized transport coefficients the following:

$$
\begin{aligned}
(\sigma=) L_{11} & =\frac{1}{\hbar a} \int_{-\infty}^{\infty}\left[-f^{\prime}(\omega)\right] e^{2} \tau(\omega) d \omega, \\
L_{12} & =\frac{1}{\hbar a} \int_{-\infty}^{\infty}\left[-f^{\prime}(\omega)\right] e \omega \tau(\omega) d \omega, \\
L_{22} & =\frac{1}{\hbar a} \int_{-\infty}^{\infty}\left[-f^{\prime}(\omega)\right] \omega^{2} \tau(\omega) d \omega .
\end{aligned}
$$

Here, $f^{\prime}$ is the derivative of the Fermi function; thus, we have the limits

$$
L_{11}(T=0)=\frac{e^{2}}{\hbar a} \tau(0), \quad L_{12}(T=0)=0, \quad L_{22}(T=0)=0,
$$

and $\tau(\omega)$ represents a generalized relaxation time defined as

$$
\begin{aligned}
\tau(\omega) & =\frac{2 \pi}{d}\left(t^{*}\right)^{2} \int_{-\infty}^{\infty} \rho_{0}(\varepsilon) \rho_{c}^{2}(\varepsilon, \omega) d \varepsilon \\
& =\frac{\left(t^{*}\right)^{2}}{\pi d}\left[\frac{\partial \operatorname{Im} D(z)}{\partial \operatorname{Im} z}-\frac{\operatorname{Im} D(z)}{\operatorname{Im} z}\right],
\end{aligned}
$$

which is to be evaluated at $z=x+i y$

$$
z= \begin{cases}\omega+i 0^{+}-\varepsilon_{c}-\Sigma_{c}\left(\omega+i 0^{+}\right) & (\mathrm{DMFT}) \\ \omega+i 0^{+}-\Sigma_{c c}^{\mathrm{CPA}}\left(\omega+i 0^{+}\right)-\Sigma_{c}^{\mathrm{CPA}}\left(\omega+i 0^{+}\right) & (\mathrm{CPA}) .\end{cases}
$$

Here, $\varepsilon_{c}$ corresponds to the component $\Sigma_{c c}^{\mathrm{CPA}}$, and $\Sigma_{c}$ and $\Sigma_{c}^{\mathrm{CPA}}$ are defined in Eqs. (4) and (24), respectively. For small $y=$ $\operatorname{Im} z$, e.g., in the Fermi-liquid regime, the approximation

$$
\tau(\omega)=\frac{\left(t^{*}\right)^{2}}{d}\left[\frac{\rho_{0}(x)}{y}+\frac{\rho_{0}^{\prime \prime}(x)}{2} y+O\left(y^{3}\right)\right]
$$

is valid. In lowest order, we have $\tau(\omega) \propto 1 / \operatorname{Im} \Sigma_{c}\left(\omega+i 0^{+}\right)$ and the linearized Boltzmann transport theory is recovered. Though we are using the full form of Eq. (44) for calculations, it is sufficient to consider the lowest order only for discussion of the properties of the transport coefficients because it is the main contribution.

\section{RESULTS FOR THE TRANSPORT PROPERTIES}

All transport calculations rely on the results for the singleparticle Green functions of the PAM. As stated in Sec. IID, we use a Gaussian model density of states [Eq. (29) with $\sqrt{2} t^{*}=10 \Gamma_{0}$ ] for the unperturbed conduction-electron system and measure energies in units of $\Gamma_{0}=100 \mathrm{meV}$. In particular, this defines a reference hybridization $V_{0}$ by $V_{0}^{2}=$ $2 t^{*} \Gamma_{0} / \sqrt{2 \pi}=10 \Gamma_{0}^{2} / \sqrt{\pi} \approx 5.64 \Gamma_{0}^{2}$.

If we assume one electron per unit cell of the volume $a^{3}$ $\left(a=10^{-10} \mathrm{~m}\right)$, the resistivity $\rho=\sigma^{-1}$ has the natural unit

$$
\sigma_{0}^{-1}=\hbar a / e^{2} \approx 41 \mu \Omega \mathrm{cm} .
$$

Note that $e L_{12} / k_{B} T L_{11}$ is dimensionless and $k_{B} / e \approx$ $-86 \mu \mathrm{V} / \mathrm{K}$. Therefore, the thermoelectric power is given in absolute units; only the scale of the temperature axis must be fixed by experiment. With our choice of $\Gamma_{0}$, it is given in units of $\frac{\Gamma_{0}}{k_{B}} \approx 1160 \mathrm{~K}$.

\section{A. Disorder on the $f$ site}

In this section, we restrict ourselves to the special case of Kondo holes, as introduced in Sec. III. In particular, the $f$ level energy of system $B$ is shifted to infinity. The hybridization $V=V_{A}=V_{B}$ is set to the reference value $V_{0}$.

\section{Resistivity}

In Fig. 4, the resistivity is displayed for $U / \Gamma_{0}=10, \varepsilon_{f}^{A}-$ $\varepsilon_{c}^{A}=-U / 2$, and various values of the concentration $c$ of system $A$. The filling is set to $n_{\text {tot }}=1.6-(1-c)$.

In the pure case, $c=1$, we reproduce the typical behavior of metallic heavy-fermion systems ${ }^{3,4,5,6}$ within our CPA-NRG treatment: a resistivity increasing with increasing $T$ for low $T$, a maximum of the order of $100 \mu \Omega \mathrm{cm}$ at a characteristic temperature $T_{\max }$, and a $\rho(T)$ (logarithmically) decreasing with increasing $T$ for $T>T_{\max }$. Note that even for $c=1$, a finite $\rho(T=0)$ is obtained for $T \rightarrow 0$. This is due to the fact that we are using a finite $\delta=10^{-3} \Gamma_{0}$ contributing to the self-energy imaginary part. In addition, the broadening of Eq. (27) and the limited accuracy of the NRG in the regime $\omega<T$ further enhance the self-energy imaginary part; for a discussion, see also Ref. 18.

In the disordered case, $c<1$, we distinguish the two regimes $T<T_{\max }(c=1)$ and $T>T_{\max }(c=1)$ to obtain a qualitative classification for all concentrations $c$. At high temperatures $T>T_{\max }$, the logarithmic gradient of $\rho(T)$ has its origin in the weak incoherent scattering of conduction electrons by the $f$ moments. With increasing concentration $1-c$ of Kondo holes, there are less scattering events. Indeed, the resistivity decreases and scales linearly with the concentration $c$ as depicted in Fig. 4(b). The $c$ dependence of the resistivity $\rho$ as well as of $\rho / c$ at a fixed temperature $T \approx 274 \mathrm{~K}>T_{\max }$ is plotted in greater detail in Fig. 5(b). 

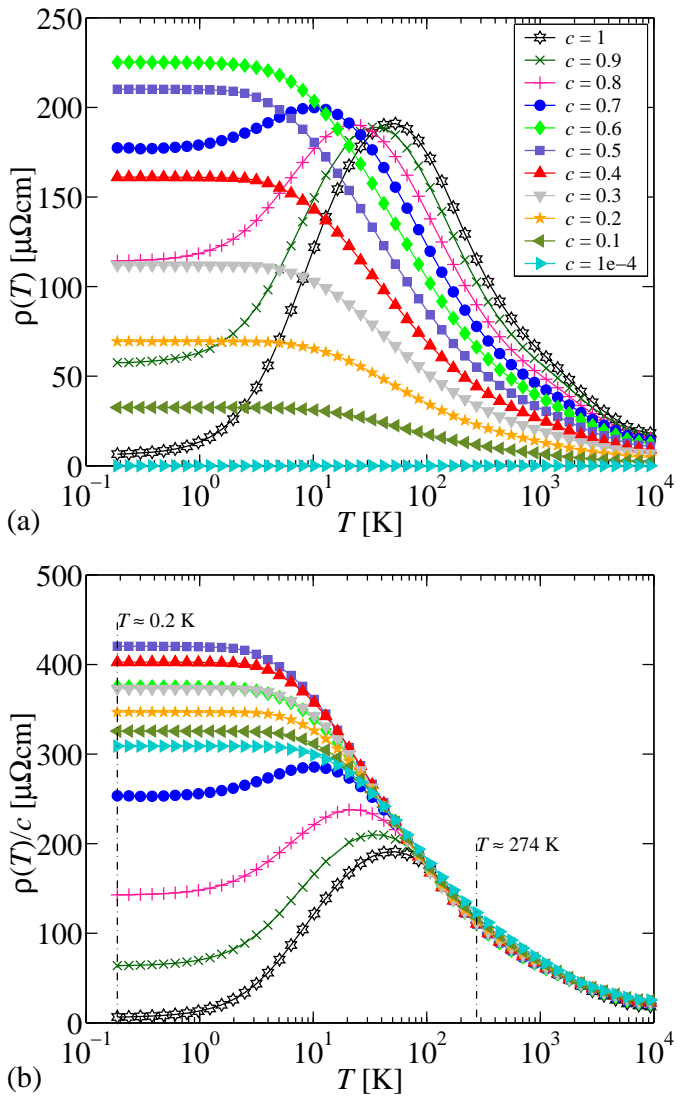

FIG. 4: (Color online) Resistivity $\rho(T)$ as a function of $T$ for different concentrations $c$ of system $A$, calculated with CPA-NRG; all parameters as in Fig. 1, i.e., $U / \Gamma_{0}=10, \varepsilon_{f}^{A}-\varepsilon_{c}^{A}=-U / 2, \varepsilon_{f}^{B}=\infty$, and $n_{\text {tot }}=1.6-(1-c)$. In (b), the same data are plotted as $\rho(T) / c$. The dash-dotted lines mark two cuts through the resistivity curves which are shown in Fig. 5 in more detail.

This can be understood using the transport equation (43a) with abbreviation (46) as follows:

$$
\frac{\rho}{c}=\sigma_{0}^{-1}\left(\int_{-\infty}^{\infty}\left[-f^{\prime}(\omega)\right] c \tau(\omega) d \omega\right)^{-1}
$$

Because $\frac{1}{c} \operatorname{Im} \Sigma_{c}(\omega)$ is nearly independent of $c$ for temperatures $T>T_{\max }$ [cf. Fig. 3(b)], also $c \tau(\omega)$ does not depend on $c$ [see Fig. 5(d)].

At temperatures $T<T_{\max }$, conduction electrons and $f$ electrons tend to form heavy quasiparticles with Fermi-liquid properties, leading to a small resistivity for $c=1$ and $T \rightarrow 0$. The introduction of Kondo holes destroys the lattice periodicity and, therefore, the resistivity increases; for $c \leq 0.5$, the nonmonotonic course changes to a behavior which is typical for a dilute distribution of impurity scattering centers [cf. Fig. 4(a)]. As a consequence, the resistivity at $T<T_{\max }$ does not scale with the concentration $c$ of Kondo holes. Instead, the normalized residual resistivity $\rho(T=0) / c$ depends on $c$ and reaches a maximum at $c=0.5$ [cf. Fig. 5(a)]; for small $c$
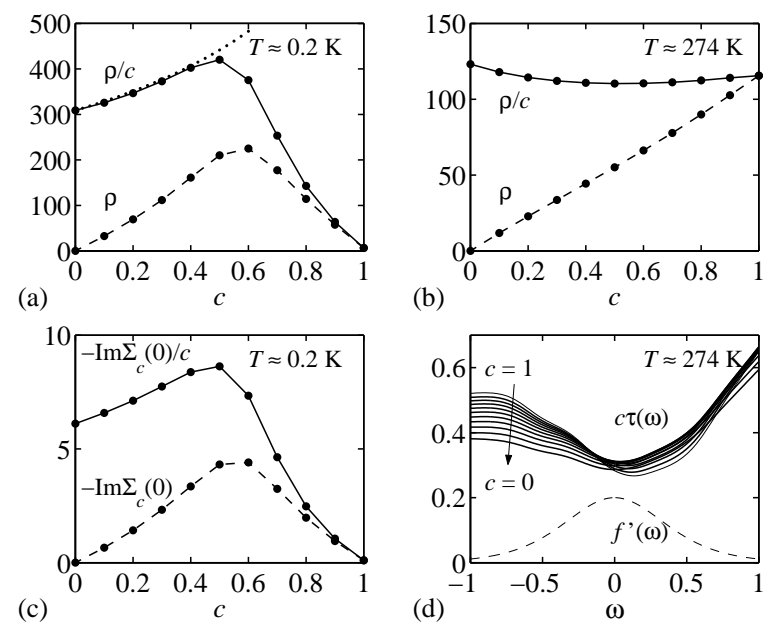

FIG. 5: [(a) and (b)] Resistivity as a function of the concentration $c$ at $T \approx 0.2 \mathrm{~K}$ and $T \approx 274 \mathrm{~K}$, respectively: $\rho(c)$ (dashed line), $\rho(c) / c$ (full line), and approximation $\propto 1 /(1-0.6 c)$ (dotted line). (c) Illustration of the proportionality $\rho(T \rightarrow 0) / c \propto \operatorname{Im} \Sigma_{c}^{\mathrm{CPA}}(0) / c$ [cf. Fig. 3]. (d) Generalized relaxation time $c \tau(\omega)$ for $T \approx 274 \mathrm{~K}$. The additional Fermi derivation shows the relevant part of the energy axis. The temperatures correspond to dash-dotted lines in Fig. 4.

it can be approximated by

$$
\frac{r_{0}}{1-0.6 c} \text { with } r_{0}:=\lim _{c \rightarrow 0} \frac{\rho(T=0)}{c} .
$$

This is consistent with other calculations ${ }^{36,38,42}$ and can be understood using the CPA equation. The approximation Eq. (45) leads to $\rho(T=0) / c=\sigma_{0}^{-1} / c \tau(0) \propto-\operatorname{Im} \Sigma_{c}^{\mathrm{CPA}}(0) / c$ [using the limit (43d) with abbreviation (46)]. With the CPA equation (31), we obtain

$$
\frac{1}{c} \operatorname{Im} \Sigma_{c}^{\mathrm{CPA}}(0)=\frac{1}{c} \operatorname{Im} \frac{V^{2}}{-\Sigma_{f f}^{\mathrm{CPA}}(0)}=\operatorname{Im} \frac{V^{2} G_{f f}^{A}(0)}{1+c \Delta_{A}(0) G_{f f}^{A}(0)} .
$$

Because $\Delta_{A}(0) G_{f}^{A}(0)$ does not vanish, $\operatorname{Im} \Sigma_{c}^{\mathrm{CPA}}(0) / c$ as well as $\rho(T=0) / c$ acquire a $1 /(1-a c)$ dependence.

A word is in order about the relation of the concentration dependence of the resistivity to the typical scaling behavior for heavy-fermion materials, e.g., in $\mathrm{Ce}_{x} \mathrm{La}_{1-x} \mathrm{Cu}_{6}{ }^{6}$ The reported magnetic resistivity contribution normalized onto the Ce concentration decreases monotonically with increasing cerium concentration. ${ }^{6}$ In $\mathrm{Ce}_{x} \mathrm{La}_{1-x} \mathrm{Cu}_{2.05} \mathrm{Si}_{2}$, however, a nonmonotonic concentration dependence of the magnetic part of the resistivity, normalized to its room temperature value, was found. ${ }^{14,15}$ Our calculations describe consistently the crossover from a concentrated lattice system to a dilute distribution of impurity scattering centers. As discussed above, we can clearly pinpoint the origin of the maximum of the normalized residual resistivity $\rho(0) / c$ at concentrations $c=x=0.5$. It arises from multiple scattering processes at intermediate concentrations, and the CPA equation yields a low-temperature enhancement factor of about $1 /(1-a c)$ [see Eq. (47)]. In the dilute limit, $\rho(0) / c$ becomes independent 


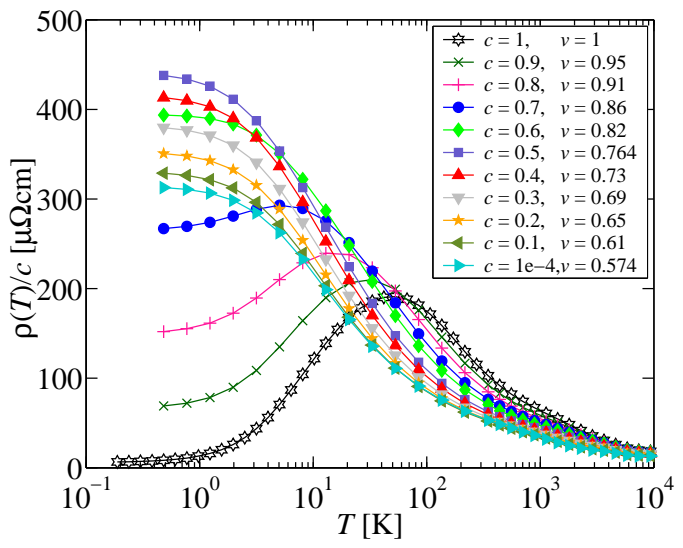

FIG. 6: (Color online) Resistivity as in Fig. 4(b), but with $c$ dependent hybridization $V^{2}=v(c) V_{0}^{2}$.

of the concentration where the conduction band self-energy is proportional to the single-particle $t$-matrix. ${ }^{99}$

A weak dependence of the low-energy scale as a function of doping has been reported in experiments. ${ }^{6,15}$ To simulate these findings, we also studied an explicitly concentrationdependent hybridization: $V^{2}$ is scaled down in such a way that the same temperature scale $T_{0}$ is obtained for all concentrations. This is achieved by a linear interpolation of $V^{2}$ between the concentrated limit and the dilute limit. Since La increases the lattice spacing ${ }^{6,15}$ of the materials, a decrease of the hybridization strength with increasing concentration $1-c$ of the Kondo holes is consistent with the experimental findings. In Fig. 6, the resistivity is displayed for the same parameters as in Fig. 4, but for $c$-dependent hybridization. As a consequence, the calculated curves for the resistivity scale with $c v(c)$. Since we do not know how to relate the explicit change of the hybridization to a change of the unit cell volume, we are not able to rescale the absolute unit.

The experimental determination of the resistivity faces some severe limitations in determining the absolute value of magnetic contribution to the resistance. Setting aside any problems stemming from the subtraction of the phonon contributions by using a proper reference material, ${ }^{6,14}$ three major sources of errors remain: (i) the accuracy of the geometry factor relating the measured resistance to the resistivity, (ii) the accuracy of the determination of the concentration $c$, and (iii) dealing with grain boundaries typical for such heavy-fermion alloys. In particular, the error of the concentration value $c$ increases significantly in the dilute limit, which has a profound impact on the absolute value of $\rho(T) / c$. In addition grain boundaries in samples might require the subtraction of the additional boundary resistivity in order to extract $\rho_{\text {mag }}(T)$ instead of normalizing the resistivity to its room temperature value, since grain boundaries are highly sample dependent. Therefore, we limit ourselves to the reproduction of the qualitative features of the reported experimental behavior.

We also investigated the influence of the local Coulomb repulsion on the transport properties. In Fig. 7, the resistivity is displayed for a fixed concentration $c=0.8, \varepsilon_{f}^{A}-\varepsilon_{c}^{A}=-U / 2$,
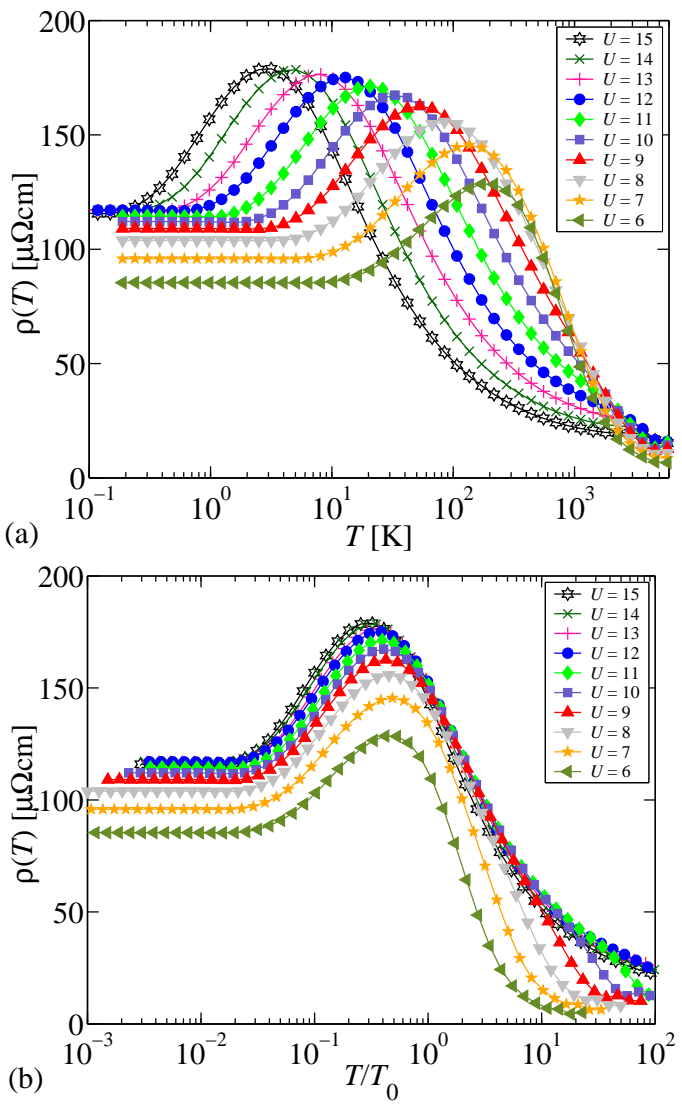

FIG. 7: (Color online) Resistivity $\rho(T)$ as a function of $T$ for concentration $c=0.8$ of system $A$, calculated with CPA-NRG for different $U / \Gamma_{0}, \varepsilon_{f}^{A}-\varepsilon_{c}^{A}=-U / 2, \varepsilon_{f}^{B}=\infty$, chemical potential $\mu=0$, and a filling $n_{\text {tot }}=1.4$. NRG parameters: number of retained NRG states, $N_{s}=800, \Lambda=1.6, \delta / \Gamma_{0}=10^{-10}$, and $L_{w}=0$ (i.e., no Lorentzian broadening).

and various values of $U / \Gamma_{0}$. The filling is set to $n_{\text {tot }}=1.4$ to match the previous setting $n_{\text {tot }}=1.6-(1-c)$ in the case of fixed $U / \Gamma_{0}=10$. Here, we reduced the small imaginary part $\delta / \Gamma_{0}$ to $10^{-10}$ to reduce the error in the residual resistivity to a minimum. While it turned out to be sufficient to use $\delta / \Gamma_{0}=10^{-3}$ for values of the Coulomb interaction up to $U / \Gamma_{0}=10$, this is no more the case for $U / \Gamma_{0}>10$.

For a particle-hole symmetric conduction band, no evenodd oscillations in the NRG spectral function have been reported ${ }^{82}$ On the contrary, we observe even-odd oscillations in the NRG spectral function of the effective site with respect to the number of NRG iterations, which is related to the temperature of the calculation in the usual way. ${ }^{22,81}$ This feature is related to the two strong coupling fixed points ${ }^{22}$ of the NRG and can show up for particle-hole asymmetric band density of states. We used the standard technique for thermodynamical properties such as entropy ${ }^{22,81}$ and average the resistivity as a function of temperature for even and odd numbers of iterations.

The temperature $T_{\max }$, at which the resistivity has its maximum, is shifted to lower values for increasing $U$; one obtains an exponential dependency of $T_{\max }$ on $U$. For sufficiently 


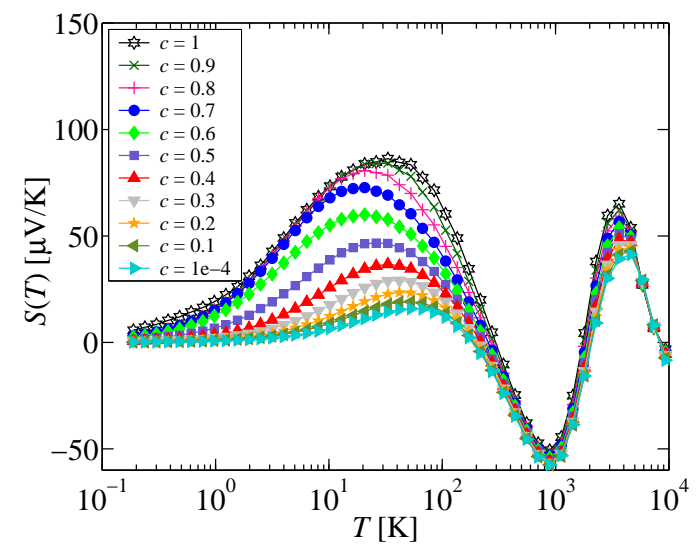

FIG. 8: (Color online) Thermoelectric power $S(T)$ as a function of $T$ for fixed $U / \Gamma_{0}=10$ and different concentrations $c$ of system $A$; all parameters as in Fig. 4, among others $n_{\text {tot }}=1.6-(1-c)$.

strong $U$, the peak height at $T_{\max }$ is nearly independent of the value of $U$. This is characteristic for the disordered stable moment regime, in which the $f$ occupation reaches integer valence, and the increase of $U$ only reduces the effective Kondo coupling $J \propto V^{2} / U$. Charge fluctuations are strongly suppressed at low temperatures, and one reaches a universal regime for a fixed concentration. This is seen more clearly from Fig. 7(b), which shows the scaling properties plotting the resistivity $\rho(T)$ versus $T / T_{0}$. We have defined $T_{0}$ in Eq. (33) as an effective low-temperature scale of subsystem $A$ in order to clarify the scaling properties of transport properties. We certainly do not imply universality of all low-energy properties. We note, however, that $T_{0}$ is of the order of the position $T_{\max }$ of the maximum of the resistivity. While for $U / \Gamma_{0}<8$, where high- and low-temperature scales are not very well separated, the maximum of the resistivity and the peak height show a $U$ dependence, and we reach a universality regime for large $U$.

\section{Thermoelectric power}

The thermoelectric power $S(T)$ measures the ratio between electrical and heat current divided by the temperature, and its sign is related to the integrated particle-hole asymmetry relative to the chemical potential. In Fig. $8, S(T)$ is plotted for fixed $U / \Gamma_{0}=10, \varepsilon_{f}^{A}-\varepsilon_{c}^{A}=-U / 2$, and various values of the concentration $c$ of system $A$. The filling is kept at $n_{\text {tot }}=1.6-(1-c)$ and the $f$-level energy of system $B$ is shifted to infinity. This figure is accompanied by Fig. 9 . Here, $S(T)$ is plotted for fixed filling $n_{\text {tot }}=1.4, c=0.8$, $\varepsilon_{f}^{A}-\varepsilon_{c}^{A}=-U / 2$, and various values of the Coulomb interaction $U$ [for the low-temperature scale $T_{0}$, see Eq. (33)].

We obtain very large absolute values for $S(T)$ (of the magnitude $100 \mu \mathrm{V} / \mathrm{K}$; see also Ref. 18). Note that the thermoelectric power is obtained in absolute units, as already mentioned in the beginning of this section. Similar to the resistivity, the thermoelectric power exhibits a low-temperature peak which is correlated with the maximum of the resistivity, which is an
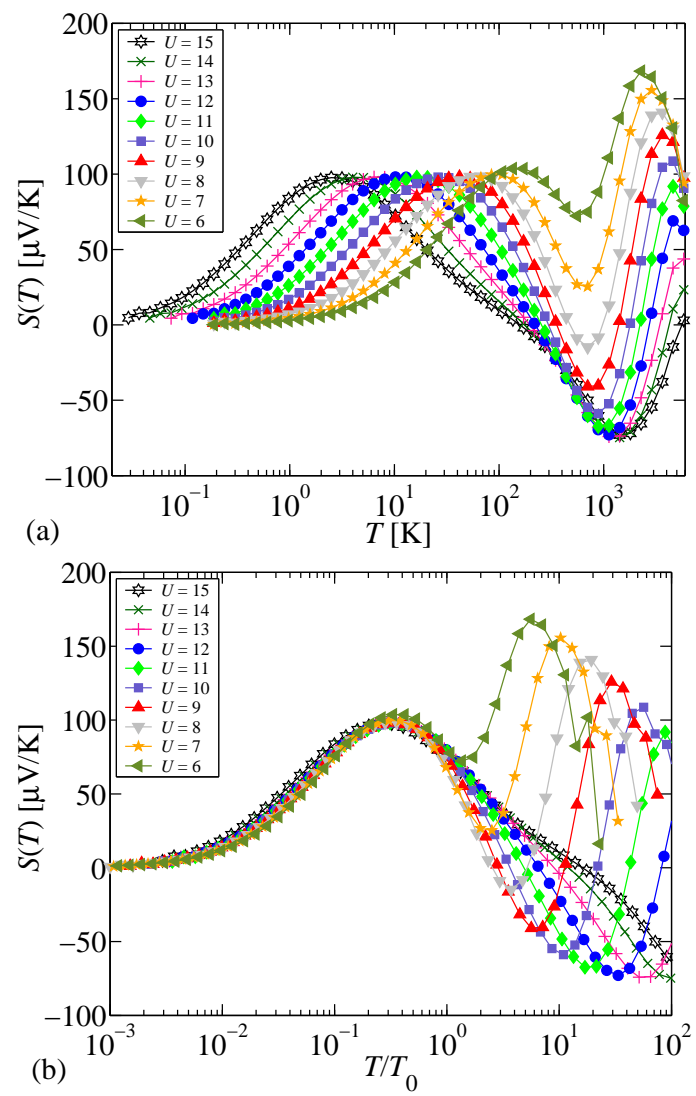

FIG. 9: (Color online) Thermoelectric power $S(T)$ as a function of $T$ for fixed concentration $c=0.8$ of system $A$ and different $U / \Gamma_{0}$; all parameters as in Fig. 7, among others $n_{\text {tot }}=1.4$.

analytical consequence of Eq. (42). Therefore, the position of this low-temperature peak depends on the low-temperature scale $T_{0}$, which varies with the concentration $c$. This can be seen in Fig. 9(b), where the thermoelectric power is shown on a rescaled axis $T / T_{0}$.

In addition, we observe a second extremum at a very high temperature independent of the concentration and the lowtemperature scale which results from the charge fluctuations on the energy scale $\varepsilon_{f}-\mu$. This maximum moves to higher temperatures with increasing $U$, as shown in Fig. 9.

\section{B. Disorder on the ligand sites}

In this section, we use the full matrix version of the CPA of Sec. IIC, where the two subsystems may have different parameter matrices $\mathscr{V}_{A}$ and $\mathscr{V}_{B}$; i.e., we introduce disorder on the ligand sites. We consider two cases: At first, we keep the same hybridization for both subsystems, $V_{A}=V_{B}=V_{0}$; afterwards, we use a site-dependent hybridization $V_{A} \neq V_{B}$. In both cases, we fix the interaction at $U=10 \Gamma_{0}$. 

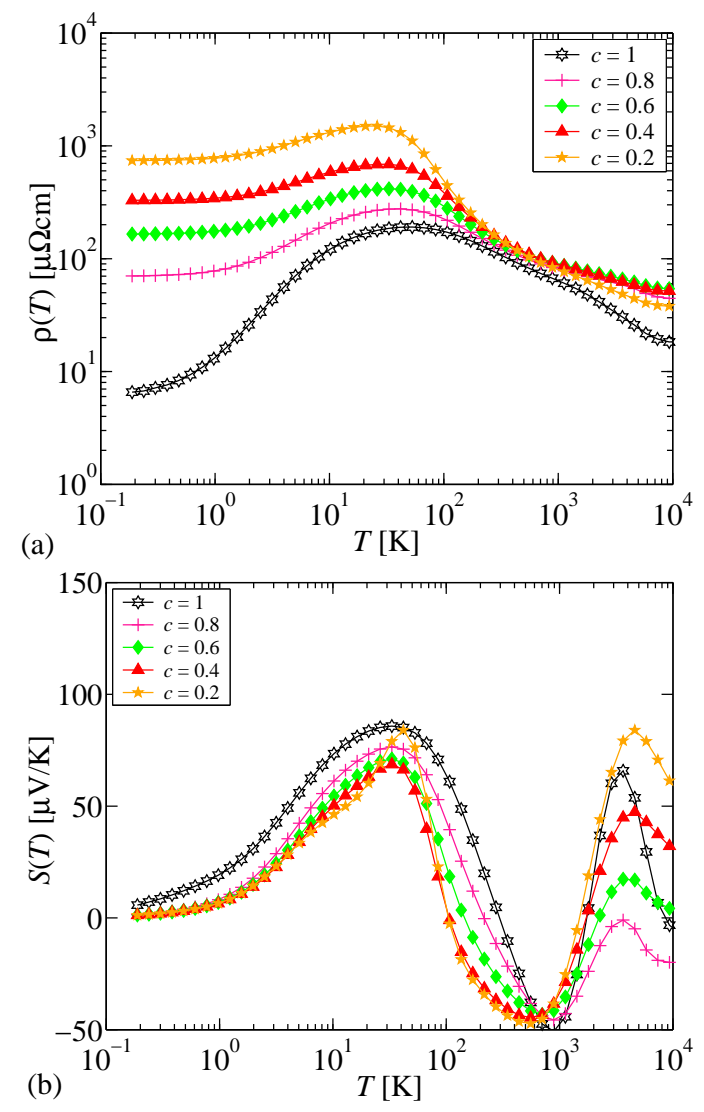

FIG. 10: (Color online) (a) Resistivity $\rho(T)$ and (b) thermoelectric power $S(T)$ as functions of $T$ for different concentrations $c$ of system $A$, calculated with CPA-NRG for $V_{A}=V_{B}=V_{0}, U / \Gamma_{0}=10, \varepsilon_{f}^{A}-$ $\varepsilon_{c}^{A}=-U / 2, \varepsilon_{f}^{B}-\varepsilon_{c}^{B}=-\Gamma_{0}, \varepsilon_{f}^{A}=\varepsilon_{f}^{B}$, chemical potential $\mu=0$, and a filling $n_{\text {tot }}=2-0.4 c$. NRG parameters: number of retained NRG states, $N_{s}=800, \Lambda=1.6$, and $\delta / \Gamma_{0}=10^{-3}$.

\section{Resistivity and thermopower for $V_{A}=V_{B}=V_{0}$}

We start with the investigation of the transport properties as a function of disorder in the case in which ligand substitution drives the system from metallic to Kondo-insulating behavior. The concentration-dependent filling is set to $n_{\text {tot }}=2-0.4 c$, and the system evolves into a Kondo insulator by reducing c. In Fig. 10, the resistivity and the thermoelectric power are displayed for $\varepsilon_{f}^{A}-\varepsilon_{c}^{A}=-U / 2, \varepsilon_{f}^{B}-\varepsilon_{c}^{B}=-\Gamma_{0}$, and various values of the concentration $c$ of system $A$. The curves for $c=1$ are identical to the $c=1$ curves in Figs. 4 and 8. We plot the resistivity on a log-log scale in Fig. 10(a) to cover the almost 3 orders of magnitude of resistivity change.

In contrast to the previous case of $f$ disorder introduced by Kondo holes, the number of Kondo scatterers remains constant as a function of the concentration $c$. Therefore, the resistivity does not vanish for $c \rightarrow 0$. For temperatures $T>T_{\max }$, the absolute values of the resistivity remain nearly the same; above this characteristic low-temperature scale $T_{\max }$, lattice and Kondo disorder scattering become indistinguishable. For temperatures $T<T_{\max }$, an increase in the resistivity with de-
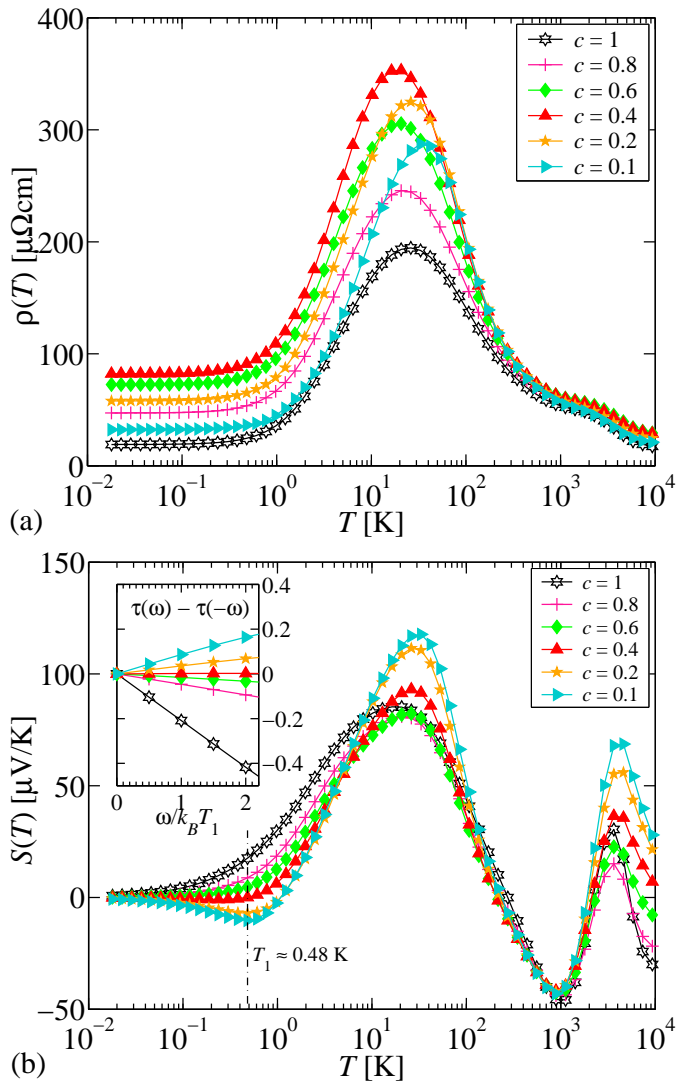

FIG. 11: (Color online) (a) Resistivity $\rho(T)$ and (b) thermoelectric power $S(T)$ as functions of $T$ for different concentrations $c$ of system $A$, calculated with CPA-NRG for $V_{A}=V_{B}=V_{0}, U / \Gamma_{0}=10$, $\varepsilon_{f}^{A}-\varepsilon_{c}^{A}=-6 \Gamma_{0}, \varepsilon_{f}^{B}-\varepsilon_{c}^{B}=-4 \Gamma_{0}, \varepsilon_{f}^{A}-\varepsilon_{f}^{B}=0.2 \Gamma_{0}$, chemical potential $\mu=0$, and a filling $n_{\text {tot }}=1.8-0.2 c$. The inset of (b) shows the antisymmetrized relaxation time $\tau(\omega)-\tau(-\omega)$ [Eq. (44)] in the vicinity of the chemical potential for the temperature $T_{1}=0.48 \mathrm{~K}$. NRG parameters: number of retained NRG states, $N_{s}=800, \Lambda=1.6$, and $\delta / \Gamma_{0}=10^{-3}$.

creasing concentration $c$ is observed; Fig. 10(a) shows a transition from low resistivity to an insulating behavior for $T \rightarrow 0$ and $c \rightarrow 0$. The disorder introduced in the conduction band destroys the lattice coherence of the heavy quasiparticles at low temperatures. Even though translational invariance is restored for $c \rightarrow 0$, the residual resistivity remains increasing for $c<0.5$ due to the crossover to an insulating behavior.

The thermoelectric power does not show much variation with the concentration $c$ as can be seen in Fig. 10(b). In contrast to the resistivity, the thermoelectric power depends on the asymmetry of the spectrum [see Eqs. (42) and (43b)]. It turns out that the asymmetry of the spectra as well as the asymmetry of the relaxation time as defined in Eq. (44) change only very weakly with the concentration, even though there is a significant renormalization of the effective media $\Delta_{A / B}(z)$ [Eq. (21)] which enters the effective site calculations.

Next, we investigate the transport properties as a function of disorder in the case in which ligand substitution keeps the system in a metallic regime for all values of $c$. In Fig. 11(a), 
the resistivity is displayed for $\varepsilon_{f}^{A}-\varepsilon_{c}^{A}=-6 \Gamma_{0}$ and $\varepsilon_{f}^{B}-\varepsilon_{c}^{B}=$ $-4 \Gamma_{0}$. In this case, we have a disordered conduction band where both subsystems $A$ and $B$ are asymmetric. By choosing a filling of $n_{\text {tot }}=1.8-0.2 c$ and $\varepsilon_{f}^{A}-\varepsilon_{f}^{B}=0.2 \Gamma_{0}$, a nearly $c$ independent $f$ occupation $n_{f} \approx 0.92$ is achieved, significantly departed from the Kondo insulator regime. This is clearly visible in the figure: in the limits $c=0$ and $c=1$, the residual resistivity $\rho(T \rightarrow 0)$ is small as expected for metallic systems. For $0<c<1$, the disorder in the conduction band leads to an enhanced resistivity. The residual resistivity peaks between $c=0.4$ and 0.6. At high temperatures, the resistivity becomes independent of the concentration $c$ : the incoherent Kondo scattering dominates the scattering processes over the lattice disorder.

In Fig. 11(b), the thermoelectric power is shown for the same parameter set. The overall appearance is equal to the case with Kondo holes, but, in addition, we observe a sign change at small temperatures. This sign change originates from the change of asymmetry in the relaxation time $\tau(\omega)$ as defined in Eq. (44). To illustrate this point, we plot the antisymmetrized relaxation time $F(\omega):=\tau(\omega)-\tau(-\omega)$ in the vicinity of the chemical potential for $T_{1}=0.48 \mathrm{~K}$ as inset in Fig. 11(b). At this temperature, $S(T, c=0.1)$ reaches its lowtemperature minimum, while for $c=1$, a positive thermoelectric power is found. For intermediate $c=0.4, F(\omega)$ almost vanishes as can be seen in the inset; simultaneously, the value of the thermoelectric power at $T_{1}$ is very small. For concentrations $c<0.4$, the positive slope of $F(\omega)$ leads to a negative thermoelectric power, while for $c>0.4$, a negative slope is found corresponding to a positive thermoelectric power. This change of asymmetry is a consequence of the very subtle redistribution of spectral weight also seen in the single particle spectra (not depicted here).

\section{Resistivity and thermopower for $V_{A} \neq V_{B}$}

In a real material, it is more likely that the hybridization changes with the local environment. Two different scenarios could be discussed. The first one considers each individual local configuration to be characterized by a probability distribution function for the hybridization $P(V)$, which will lead to a distribution of Kondo scales. This scenario was suggested as a possible route to non-Fermi-liquid behavior in HFSs. ${ }^{44}$ We, however, restrict ourselves to $A / B$ ligand disorder, yielding one effective hybridization for each type of lattice sites $A$ and $B: V_{A}$ and $V_{B}$. For a homogeneous medium $\Gamma$ as defined in Eq. (20), this leads to two different energy scales $T_{A}$ and $T_{B}$ for the local effective sites $A$ and $B$. We expect a crossover from one heavy fermion with a lower characteristic energy scale to another with a higher value of $T_{0}$.

We take Fig. 11 as reference and adapt the choice of parameters to it. There are two possibilities to achieve $V_{A} \neq V_{B}$ : we can change $V_{A}$ or $V_{B}$ with respect to Fig. 11. Both cases are discussed in the following. We reduced the small imaginary part $\delta / \Gamma_{0}$ to $10^{-4}$ in order to reduce the error in the residual resistivity. In Fig. 12, the resistivity and the thermoelectric power for ligand disorder with changing hybridiza-
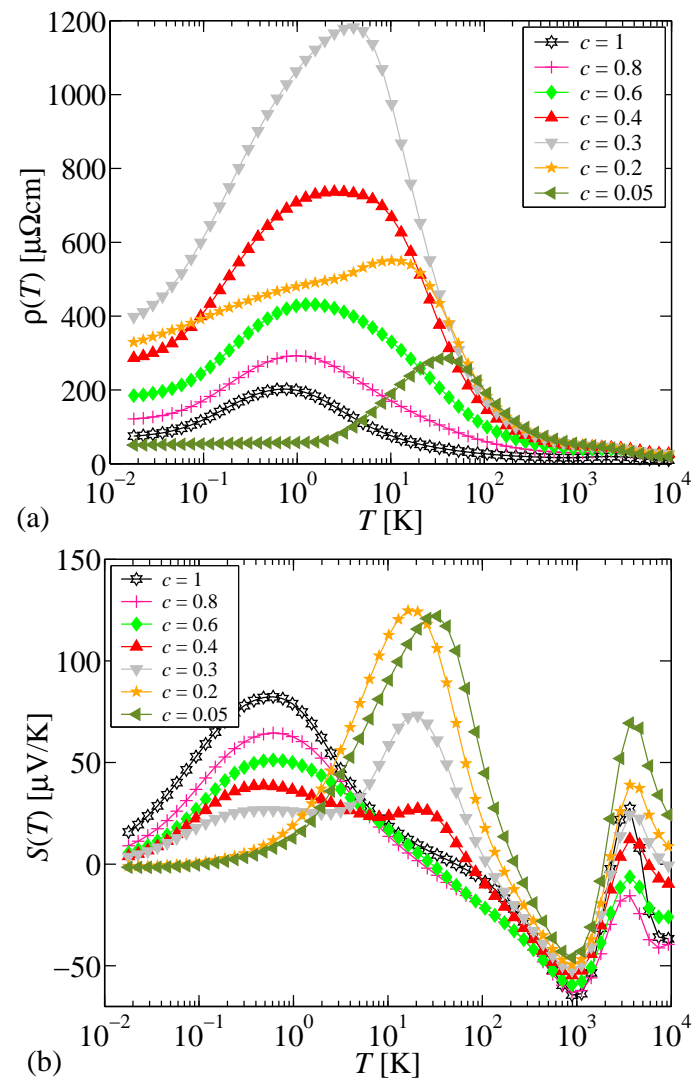

FIG. 12: (Color online) (a) Resistivity $\rho(T)$ and (b) thermoelectric power $S(T)$ as functions of $T$ for different concentrations $c$ of system $A$; all parameters as in Fig. 11, except for $V_{A}^{2}=3 \Gamma_{0}^{2}$ and $\delta / \Gamma_{0}=$ $10^{-4}$.

tion from a low value of $V_{A}^{2}=3 \Gamma_{0}^{2}$ at $c=1$ to a high value of $V_{B}^{2}=V_{0}^{2} \approx 5.64 \Gamma_{0}^{2}$ are shown; in Fig. 13, the hybridization conversely changes from a high value of $V_{A}^{2}=V_{0}^{2} \approx 5.64 \Gamma_{0}^{2}$ at $c=1$ to a low value of $V_{B}^{2}=3 \Gamma_{0}^{2}$. All other parameters are the same as in Fig. 11.

In both figures, the CPA end points $c=0$ and $c=1$ nicely illustrate the different energy scales, which are proportional to the temperature value of the resistivity maximum and the corresponding maximum of the thermoelectric power. With varying concentration, the thermoelectric power reflects the crossover from one energy scale to the other by a decreasing height of one maximum and an increasing height of the other one. At intermediate concentrations, two maxima in $S(T)$ are found, which clearly shows the presence of two energy scales. This feature is most pronounced in Fig. 12 for $c=0.4$, where a shallow double maximum structure is observed.

The behavior of the resistivity is completely different in the two cases of Figs. 12 and 13. In Fig. 12, the residual resistivity increases with decreasing $c$ and peaks around $c=0.3$. Due to the change of particle numbers with the concentration, the residual resistivity is not symmetric around $c=0.5$. At intermediate concentrations, we find an extended crossover regime. At $c=0.2$, a non-Fermi-liquid behavior of the resistivity is observed over more than three decades in temperature, 

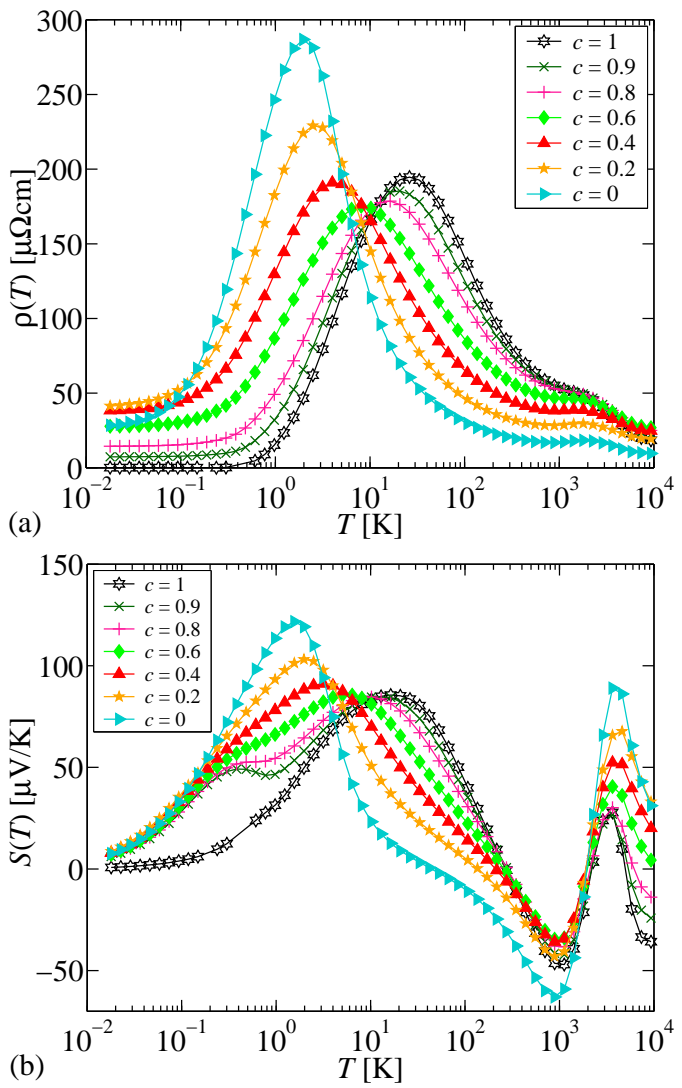

FIG. 13: (Color online) (a) Resistivity $\rho(T)$ and (b) thermoelectric power $S(T)$ as functions of $T$ for different concentrations $c$ of system $A$; all parameters as in Fig. 11, except for $V_{B}^{2}=3 \Gamma_{0}^{2}$ and $\delta / \Gamma_{0}=$ $10^{-4}$.

while the thermoelectric power is already dominated from the $B$ site transport. Although the $c=0$ curves in Fig. 13 have the same hybridization as the $c=1$ curves in Fig. 12, they differ in the number of electrons per unit cell, since in both cases $n_{\text {tot }}$ is given by $n_{\text {tot }}=1.8-0.2 c$. The change in the resistivity as a function of concentration appears to be much more gradually with a continuous and monotonic shift of the position of the resistivity maximum.

\section{CONCLUSION AND OUTLOOK}

We have presented a detailed analysis of the transport properties of disordered heavy-fermion compounds based on a combination of CPA to treat the disorder and DMFT to handle the correlation effects. Our approach reduces to the standard DMFT at those points corresponding to concentrated systems ( $c=0$ and $c=1$ ); i.e., our treatment of the transport properties interpolates between two concentrated systems consisting only of pure $A$ or $B$ sites.

To make contact to typical experimental situations, we have studied two types of local disorder: introduction of Kondo holes and disorder on the ligand sites. In the case of doping with Kondo holes, we find an increasing charac- teristic low-temperature scale $T_{0}$, defined via the quasiparticle renormalization factor, as a function of the hole concentration for constant hybridization. This is consistent with a previous comparison between the DMFT and SIAM energy scales. ${ }^{18,24}$ However, in experiments, often only a weak dependence of the low-energy scale as a function of doping has been observed. ${ }^{6,15}$ Therefore, we investigated the change of the transport properties as a function of concentration for a fixed effective low-energy scale. Such an ansatz is consistent with an experimentally found increase of the lattice constants upon Ce substitution by La, justifying a linear reduction of the hybridization strength with increasing concentration of the Kondo holes.

The calculated resistivity normalized to the Ce concentration shows a characteristic logarithmic upturn when lowering the temperature. In the periodic system, the resistivity decreases again after a maximum is reached, which is a sign of lattice coherence. Introducing Kondo holes reduces the scattering of the $f$ electrons and gradually destroys lattice coherence. The normalized resistivity continuously varies from that of a concentrated system to that of a typical dilute Kondo scatterer. While we observe scaling with the concentration at high temperature, multiscattering of conduction-band electrons introduces an additional enhancement factor $1 /(1-a c)$ in the residual resistivity for $c \rightarrow 0$, which can become already important for concentrations less than $10 \%$. Since the thermoelectric power is given by a ratio of two transport integrals, it is much less sensitive to disorder.

The scaling properties of doped and undoped heavyfermion materials appear to be inconsistent with a simple Fermi-liquid theory and have caused a lot of attention in recent years. The terminology "non-Fermi liquid" was coined for those materials whose thermodynamic and transport properties are not well understood-for a review, see Ref. 1. Usually, the deviation from a $T^{2}$ law has been attributed to scattering of conduction electrons on thermal and quantum fluctuations of the magnetic order parameter in the vicinity of a quantum critical point. Such effects would generate a $k$-dependent self-energy which is not included in our treatment. The effective site for the Kondo-hole disorder problem reaches the strong-coupling fixed point at low enough temperature in our CPA-NRG theory. Consequently, we know analytically that the local self-energy $\Sigma^{A}(z)$ has Fermi-liquid properties in this regime. The scaling regime for such a $T^{2}$ law, however, turns out to be very small. The self-consistency condition and multiscattering contributions to transport properties reorganize spectral weight in our many-body calculation. Therefore, the crossover regime starts relatively early. In this regime, one might be able to identify a rather extended temperature range in which a power law with a fixed exponent $\alpha<2$ can be obtained with the same accuracy as determined in experiments. However, the physical meaning of such an exponent obtained in a crossover from a low-temperature scaling regime is not apparent to us. Thus, we did not intend to extract such a parameter.

In the case of ligand disorder, we have shown calculations which interpolate between two metallic systems as well as describe the crossover from a Kondo insulator to a metallic 
HFS. Each unit cell contains one Kondo scatterer which experiences a different environment. A changing hybridization matrix element leads to two different local low-temperature scales. For a constant hybridization matrix element, we do not observe a shift in the position of the resistivity maximum. The resistivity follows the typical heavy-fermion behavior with a concentration-dependent residual resisitivity which peaks close to the concentration of $c=0.5$. In the thermoelectric power, however, we note a sign change at very low temperatures from positive values for $c<0.5$ to negative values for $c=0.9$ similar to the one observed ${ }^{15}$ in $\mathrm{CeCu}_{2} \mathrm{Si}_{2}$. The thermoelectric power is very sensitive to particle-hole asymmetries in the spectral functions.

Our results clearly demonstrate that the combination of CPA and DMFT is capable of accurately capturing the interplay of effects that are connected to local correlations, on the one hand, and the average influence of disorder, on the other hand. We discuss ligand disorder in addition to the conventionally investigated introduction of Kondo holes. Such ligand disorder is relevant for a variety of HFSs and, in particular, interesting in connection with $\mathrm{CeCu}_{6-x} \mathrm{Au}_{x}$, the paradigm of non-Fermi-liquid behavior in the vicinity of a quantum phase transition. Due to the lack of nonlocal fluctuations in the DMFT-CPA treatment, it will not be possible to study the quantum critical regime within our method. However, within our approach0 we can investigate the influence of the ligand disorder on magnetic properties to obtain the magnetic phase diagram. Another aspect is the role of orbital degrees of freedom, in particular, on thermoelectric properties of HFSs. Again, the method introduced here is valid for such an extended model, too, but the calculations become much more involved. Investigations along these lines are in progress.

\section{Acknowledgments}

We thank R. Bulla, J. Freericks, N. Grewe, A. Hewson, U. Köhler, D. Logan, N. Oeschler, and V. Zlatić for numerous discussions, and we thank the KITP in Santa Barbara for its hospitality. C.G., F.B.A., and G.C. acknowledge financial support by the Deutsche Forschungsgemeinschaft, Project No. AN 275/5-1, and funding of the NIC, Forschungszentrum Jülich, Project No. HHB000. This research was also supported in part by the National Science Foundation under Grant No. PHY05-51164.
1 G. R. Stewart, Rev. Mod. Phys. 73, 797 (2001).

2 N. Grewe and F. Steglich, in Handbook on the Physics and Chemistry of Rare Earths, edited by K. A. Gschneidner, Jr. and L. Eyring (North-Holland, Amsterdam, 1991), Vol. 14, Chap. 97, pp. 343-474.

3 P. Scoboria, J. E. Crow, and T. Mihalisin, J. Appl. Phys. 50, 1895 (1979).

4 K. Andres, J. E. Graebner, and H. R. Ott, Phys. Rev. Lett. 35, 1779 (1975).

5 H. R. Ott, H. Rudigier, Z. Fisk, and J. L. Smith, Physica B \& C 127, 359 (1984).

6 Y. Ōnuki and T. Komatsubara, J. Magn. Magn. Mater. 63-64, 281 (1987).

7 L. Degiorgi, Rev. Mod. Phys. 71, 687 (1999).

8 Z. Fisk, J. L. Sarrao, J. D. Thompson, D. Mandrus, M. F. Hundley, A. Miglori, B. Bucher, Z. Schlesinger, G. Aeppli, E. Bucher, et al., Physica B 206-207, 798 (1995).

9 Z. Fisk, J. L. Sarrao, S. L. Cooper, P. Nyhus, G. S. Boebinger, A. Passner, and P. C. Canfield, Physica B 223\&224, 409 (1996).

10 N. S. Vidhyadhiraja, V. E. Smith, D. E. Logan, and H. R. Krishnamurthy, J. Phys.: Condens. Matter 15, 4045 (2003).

11 C. D. W. Jones, K. A. Regan, and F. J. DiSalvo, Phys. Rev. B 58, 16057 (1998).

12 C. D. W. Jones, K. A. Regan, and F. J. DiSalvo, Phys. Rev. B 60, 5282 (1999).

13 S. Burdin and P. Fulde, Phys. Rev. B 76, 104425 (2007).

14 M. Očko, B. Buschinger, C. Geibel, and F. Steglich, Physica B 259-261, 87 (1999).

15 M. Očko, Dj. Drobac, B. Buschinger, C. Geibel, and F. Steglich, Phys. Rev. B 64, 195106 (2001).

16 P. de V. du Plessis, A. M. Strydom, R. Troć, T. Cichorek, Cz. Marucha, and R. P. Gers, J. Phys.: Condens. Matter 11, 9775 (1999).

17 C. M. Varma and Y. Yafet, Phys. Rev. B 13, 2950 (1976).
18 C. Grenzebach, F. B. Anders, G. Czycholl, and Th. Pruschke, Phys. Rev. B 74, 195119 (2006).

19 Th. Pruschke, M. Jarrell, and J. K. Freericks, Adv. Phys. 44, 187 (1995).

20 A. Georges, G. Kotliar, W. Krauth, and M. J. Rozenberg, Rev. Mod. Phys. 68, 13 (1996).

21 P. W. Anderson, Phys. Rev. 124, 41 (1961).

22 K. G. Wilson, Rev. Mod. Phys. 47, 773 (1975).

23 H. R. Krishna-murthy, J. W. Wilkins, and K. G. Wilson, Phys. Rev. B 21, 1003 (1980); 21, 1044 (1980).

24 Th. Pruschke, R. Bulla, and M. Jarrell, Phys. Rev. B 61, 12799 (2000).

25 R. J. Elliott, J. A. Krumhansl, and P. L. Leath, Rev. Mod. Phys. 46, 465 (1974).

26 F. Yonezawa, in The Structure and Properties of Matter, edited by T. Matsubara (Springer, Berlin, Heidelberg, New York, 1982), vol. 28 of Springer Series in Solid-State Sciences, chap. 11, pp. 383-432, rev. transl. of the orig. japanese edition: bussei i, rev. 2nd ed.

27 V. Janiš and D. Vollhardt, Phys. Rev. B 46, 15712 (1992).

28 R. Vlaming and D. Vollhardt, Phys. Rev. B 45, 4637 (1992).

29 V. Janiš, M. Ulmke, and D. Vollhardt, Europhys. Lett. 24, 287 (1993).

30 M. Ulmke, V. Janiš, and D. Vollhardt, Phys. Rev. B 51, 10411 (1995).

31 P. J. H. Denteneer, M. Ulmke, R. T. Scalettar, and G. T. Zimanyi, Physica A 251, 162 (1998).

32 K. Byczuk, W. Hofstetter, and D. Vollhardt, Phys. Rev. B 69, 045112 (2004).

33 H. J. Leder and G. Czycholl, Z. Phys. B: Condens. Matter 35, 7 (1979).

34 G. Czycholl and H. J. Leder, Z. Phys. B: Condens. Matter 44, 59 (1981).

35 A. Yoshimori and H. Kasai, Solid State Commun. 58, 259 (1986). 
36 W. Xu and Zh.-Zh. Li, J. Phys.: Condens. Matter 2, 109 (1990).

37 R. Freytag and J. Keller, Z. Phys. B: Condens. Matter 80, 241 (1990).

38 Zh.-Zh. Li and Y. Qiu, Phys. Rev. B 43, 12906 (1991).

39 P. Schlottmann, Phys. Rev. B 46, 998 (1992).

40 S. Wermbter, K. Sabel, and G. Czycholl, Phys. Rev. B 53, 2528 (1996).

41 T. Mutou, Phys. Rev. B 64, 165103 (2001).

42 T. Mutou, Phys. Rev. B 64, 245102 (2001).

43 R. K. Kaul and M. Vojta, Phys. Rev. B 75, 132407 (2007).

44 V. Dobrosavljević, T. R. Kirkpatrick, and G. Kotliar, Phys. Rev. Lett. 69, 1113 (1992).

45 E. Miranda, V. Dobrosavljević, and G. Kotliar, J. Phys.: Condens. Matter 8, 9871 (1996).

46 E. Miranda, V. Dobrosavljević, and G. Kotliar, Phys. Rev. Lett. 78, 290 (1997).

47 A. Chattopadhyay and M. Jarrell, Phys. Rev. B 56, R2920 (1997).

48 F. Steglich, J. Aarts, C. D. Bredl, W. Lieke, D. Meschede, W. Franz, and H. Schäfer, Phys. Rev. Lett. 43, 1892 (1979).

49 N. Grewe and B. Welslau, Solid State Commun. 65, 437 (1988).

50 N. Grewe, Solid State Commun. 66, 1053 (1988).

51 B. Welslau and N. Grewe, Ann. Phys. (Leipzig) 1, 214 (1992).

52 I. N. Karnaukhov, Phys. Rev. B 56, R4313 (1997).

53 M. Jarrell, H. Pang, and D. L. Cox, Phys. Rev. Lett. 78, 1996 (1997).

54 A. N. Tahvildar-Zadeh, M. Jarrell, and J. K. Freericks, Phys. Rev. B 55, R3332 (1997).

55 R. Doradziński and J. Spałek, Phys. Rev. B 56, R14239 (1997).

56 R. Doradziński and J. Spałek, Phys. Rev. B 58, 3293 (1998).

57 F. B. Anders, Phys. Rev. Lett. 83, 4638 (1999).

58 F. B. Anders, Eur. Phys. J. B 28, 9 (2002).

59 J. A. Hertz, Phys. Rev. B 14, 1165 (1976).

60 A. J. Millis, Phys. Rev. B 48, 7183 (1993).

61 S. Sachdev, Quantum Phase Transitions (Cambridge University Press, Cambridge England, 2001).

62 N. Grewe, Z. Phys. B: Condens. Matter 52, 193 (1983).

63 Y. Kuramoto, Z. Phys. B: Condens. Matter 53, 37 (1983).

64 N. E. Bickers, Rev. Mod. Phys. 59, 845 (1987).

65 H. Keiter and J. C. Kimball, Int. J. Magn. 1, 233 (1971).

66 Y. Kuramoto, in Theory of Heavy Fermions and Valence Fluctuations, edited by T. Kasuya and T. Saso (Springer, Berlin, Heidelberg, New York, 1985), vol. 62 of Springer Series in Solid-State Sciences, pp. 152-162, proceedings of the Eighth Taniguchi Symposium.

67 C. I. Kim, Y. Kuramoto, and T. Kasuya, Solid State Commun. 62, 627 (1987).

68 C. I. Kim, Y. Kuramoto, and T. Kasuya, J. Phys. Soc. Jpn. 59, 2414 (1990).

69 N. Grewe, Z. Phys. B: Condens. Matter 67, 323 (1987).
70 N. Grewe, Th. Pruschke, and H. Keiter, Z. Phys. B: Condens. Matter 71, 75 (1988).

71 U. Brandt and C. Mielsch, Z. Phys. B: Condens. Matter 75, 365 (1989).

72 W. Metzner and D. Vollhardt, Phys. Rev. Lett. 62, 324 (1989); 62 , 1066(E) (1989).

73 E. Müller-Hartmann, Z. Phys. B: Condens. Matter 76, 211 (1989).

74 M. Jarrell, Phys. Rev. Lett. 69, 168 (1992).

75 A. Georges and G. Kotliar, Phys. Rev. B 45, 6479 (1992).

76 H. Schweitzer and G. Czycholl, Solid State Commun. 74, 735 (1990).

77 H. Schweitzer and G. Czycholl, Z. Phys. B: Condens. Matter 83, 93 (1991).

78 D. L. Cox and N. Grewe, Z. Phys. B: Condens. Matter 71, 321 (1988).

79 V. Janiš and D. Vollhardt, Phys. Rev. B 63, 125112 (2001).

80 R. Bulla, Th. Pruschke, and A. C. Hewson, J. Phys.: Condens. Matter 9, 10463 (1997).

81 R. Bulla, Th. Costi, and Th. Pruschke (2007), cond-mat/0701105, Rev. Mod. Phys. (to be published).

82 R. Peters, Th. Pruschke, and F. B. Anders, Phys. Rev. B 74, 245114 (2006).

83 A. Weichselbaum and J. von Delft, Phys. Rev. Lett. 99, 076402 (2007).

84 F. B. Anders and A. Schiller, Phys. Rev. Lett. 95, 196801 (2005).

85 F. B. Anders and A. Schiller, Phys. Rev. B 74, 245113 (2006).

86 R. Bulla, T. A. Costi, and D. Vollhardt, Phys. Rev. B 64, 045103 (2001).

87 F. B. Anders and G. Czycholl, Phys. Rev. B 71, 125101 (2005).

88 R. Bulla, A. C. Hewson, and Th. Pruschke, J. Phys.: Condens. Matter 10, 8365 (1998).

89 N. S. Vidhyadhiraja and D. E. Logan, Eur. Phys. J. B 39, 313 (2004).

90 G. Mahan, Many-Particle Physics (Plenum, New York, 1981).

91 J. M. Luttinger, Phys. Rev. 135, A1505 (1964).

92 P. Voruganti, A. Golubentsev, and S. John, Phys. Rev. B 45, 13945 (1992).

93 G. Czycholl, Theoretische Festkörperphysik (Springer, Heidelberg, 2000).

94 A. Khurana, Phys. Rev. Lett. 64, 1990 (1990).

95 H. Schweitzer and G. Czycholl, Phys. Rev. Lett. 67, 3724 (1991).

96 A. Lorek, N. Grewe, and F. B. Anders, Solid State Commun. 78, 167 (1991).

97 F. B. Anders and D. L. Cox, Physica B 230-232, 441 (1997).

98 Th. Pruschke, D. L. Cox, and M. Jarrell, Europhys. Lett. 21, 593 (1993); Phys. Rev. B 47, 3553 (1993).

99 N. E. Bickers, D. L. Cox, and J. W. Wilkins, Phys. Rev. B 36, 2036 (1987); 38, 825(E) (1988). 